\title{
Magnetic properties of G-band bright points in a sunspot moat ${ }^{\star}$
}

\author{
C. Beck ${ }^{1}$, L. R. Bellot Rubio ${ }^{2,1}$, R. Schlichenmaier ${ }^{1}$, and P. Sütterlin ${ }^{3}$ \\ 1 Kiepenheuer-Institut für Sonnenphysik, Schöneckstr. 6, 79104 Freiburg, Germany \\ e-mail: [cbeck, schliche]@kis.uni-freiburg.de \\ 2 Instituto de Astrofísica de Andalucía (CSIC), Aptdo. 3004, 18080 Granada, Spain \\ e-mail: lbellot@iaa.es \\ 3 Sterrekundig Instituut, Utrecht University, Postbus 80 000, 3508 TA Utrecht, The Netherlands \\ e-mail: P.Suetterlin@astro.uu.nl
}

Received 16 May 2006 / Accepted 6 June 2007

\section{ABSTRACT}

\begin{abstract}
We present simultaneous spectropolarimetric observations of four visible $(630 \mathrm{~nm})$ and three infrared $(1565 \mathrm{~nm})$ spectral lines from the German Vacuum Tower Telescope, together with speckle-reconstructed filtergrams in the G-band and the Ca II $\mathrm{H}$ line core from the Dutch Open Telescope. After alignment of the data sets, we used the G-band intensity to locate bright points (BPs) in the moat of a regular sunspot. With the cospatial and cotemporal information provided by the polarimetric data, we characterize the magnetic, kinematic, and thermal properties of the BPs. We find that (a) 94\% of the BPs are associated with magnetic fields; (b) their field strengths range between 500 and $1400 \mathrm{G}$, with a rather flat distribution; (c) the contrast of BPs in the G-band depends on the angle between the vector magnetic field and the line of sight; (d) the BPs harbor downflows of magnetized plasma and exhibit Stokes $V$ profiles with large area and amplitude asymmetries; (e) the magnetic interior of BPs is hotter than the immediate field-free surroundings by about $1000 \mathrm{~K}$ at equal optical depth; and (f) the mean effective diameter of BPs in our data set is $150 \mathrm{~km}$, with very few BPs larger than $300 \mathrm{~km}$. Most of these properties can be explained by the classical magnetic flux tube model. However, the wide range of BP parameters found in this study indicates that not all G-band BPs are identical to stable long-lived flux tubes or sheets of kG strength.
\end{abstract}

Key words. Sun: magnetic fields - techniques: polarimetric

\section{Introduction}

When observed at sufficient angular resolution, the solar photosphere shows small elements that appear bright in continuum intensity. Their contrast is particularly high in the G-band, the wavelength region around $430 \mathrm{~nm}$ dominated by absorption lines of the temperature-sensitive $\mathrm{CH}$ molecule, and other molecular bands such as the $\mathrm{CN}$ band head at $388 \mathrm{~nm}$ (Zakharov et al. 2005; Uitenbroek \& Tritschler 2006). The discovery of these G-band bright points (BPs) dates back to Muller (1983), although they can be identified with the "bright points" or "filigree" described earlier by Dunn \& Zirker (1973) and Mehltretter (1974).

The BPs are observed preferentially along intergranular lanes, both in active regions and in the quiet Sun. Today it is believed that they trace magnetic flux concentrations. The relationship between BPs and small-scale magnetic fields has been established from observations (Keller 1992; Berger \& Title 2001), magneto-hydrodynamical simulations (Schüssler et al. 2003; Carlsson et al. 2004; Shelyag et al. 2004), or semi-empirical models of flux concentrations (Steiner et al. 2001; Sánchez Almeida et al. 2001a). Other studies have concentrated on the evolution of BPs (Berger \& Title 1996; van Ballegooijen et al. 1998; Bovelet \& Wiehr 2003; Bonet et al. 2005) and on their spectral signatures (Langhans et al. 2002, 2004).

Despite these advances, the magnetic field strength and other physical properties of the G-band BPs remain largely unknown. This is because most studies have used narrow-band filtergrams and longitudinal magnetograms, which offer high spatial

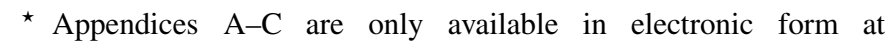
http://www . aanda.org resolution but do not allow the determination of the three components of the vector magnetic field. In this paper we investigate the magnetic, kinematic, and thermal properties of G-band BPs in the moat of a regular spot using simultaneous vector polarimetric measurements of four visible and three infrared lines, complemented with diffraction-limited narrow-band filtergrams. We also characterize the physical properties of the outer part of the moat and surrounding quiet Sun, which do not show G-band brightness enhancements. Our aim is to provide a complete observational picture of the vector magnetic fields of a large sample of BPs to help understand their nature and origin. The results of this study may be useful for validating theoretical models and MHD simulations of magnetic flux concentrations in the solar photosphere, as well as to confirm the mechanisms by which G-band bright points are generated.

The multi-wavelength observations used here have become possible only very recently, so we give a detailed account of the reduction process and the spatial alignment of the data sets in Sect. 2. Our study is based on line parameters extracted from the observed Stokes profiles (Sect. 3.2), as well as on the inversion of the visible and infrared lines (Sect. 3.3). In Sect. 4 we present the main results of a statistical analysis of the field strength, field inclination, magnetic flux, velocity, temperature, and size of BPs. Our findings are discussed in Sect. 5 and summarized in Sect. 6.

\section{Observations and data reduction}

We observed the sunspot NOAA 10425 on August 9, 2003 from 09:36 to 10:34 UT with the two spectropolarimeters of the 
Table 1. Spectral lines observed at the VTT.

\begin{tabular}{lccccr}
\hline \hline Instrument & Species & $\lambda_{0}[\mathrm{~nm}]$ & $\log g f$ & $g_{\text {eff }}$ & Pixel size \\
\hline TIP & Fe I & 1564.7410 & -0.950 & 1.25 & 0.35 \\
TIP & Fe I & 1564.8515 & -0.669 & 3.00 & \\
TIP & Fe I & 1565.2874 & -0.095 & 1.45 & \\
\hline POLIS vis & Fe I & 630.15012 & -0.750 & 1.67 & $0{ }^{\prime \prime} 145$ \\
POLIS vis & Fe I & 630.24936 & -1.236 & 2.50 & \\
POLIS vis & Fe I & 630.34600 & -2.550 & 1.50 & \\
POLIS vis & Ti I & 630.37525 & -1.444 & 0.92 & \\
\hline POLIS UV & Ca II & 396.849 & & & $0 !^{\prime} 292$ \\
\hline
\end{tabular}

German Vacuum Tower Telescope (VTT) at the Observatorio del Teide (Tenerife, Spain): the Tenerife Infrared Polarimeter (TIP; Martínez Pillet et al. 1999) and the Polarimetric Littrow Spectrograph (POLIS; Schmidt et al. 2003; Beck et al. 2005b). Both instruments were used simultaneously. The same sunspot was observed between 08:25 and 11:58 UT with the Dutch Open Telescope (DOT) at the Observatorio del Roque de los Muchachos (La Palma, Spain). The sunspot was located at an heliocentric angle of $27^{\circ}$.

\subsection{Observational setup at the VTT}

TIP uses the main spectrograph of the VTT for observations in the infrared (IR), while POLIS is a stand-alone spectropolarimeter with two channels. One records the pair of $\mathrm{Fe} I$ lines at $630 \mathrm{~nm}$, and the other measures the Ca II H line at $396 \mathrm{~nm}$. In the following, the two POLIS channels will be referred to as "visible" and "UV" channels, respectively.

An achromatic 50-50 beam splitter was used to split the light between TIP and POLIS. Scanning of the solar surface was performed with the tip-tilt mirror of the correlation tracker system (CT; Schmidt \& Kentischer 1995; Ballesteros et al. 1996), which affects the FOV of both instruments in the same way. The CT also provided image stabilization, allowing us to reach a spatial resolution of about $1^{\prime \prime}$, as derived from the power spectrum of a granulation area (cf. Appendix A). Both TIP and POLIS measure the polarization state by modulation of the incoming light with ferro-electric liquid crystals and a rotating waveplate, respectively. To observe the same field of view (FOV) with the two instruments, we aligned the TIP and POLIS slits by rotating the VTT main spectrograph to the POLIS slit orientation. The lateral displacement of the images due to the beam splitter was corrected for with the scan mirror of POLIS.

The slit width was 0.35 for TIP and $0 . ' 48$ for POLIS. The FOV along the slit was $34^{\prime \prime}$ for TIP and $47^{\prime \prime}$ for the visible channel of POLIS. The step of the spatial scan was 0.35 , with a total integration time of 6 seconds per scan step. The observations presented here consist of eight scans of 70 steps (24". 5) over the limb side part of the sunspot and its surroundings. The cadence for the VTT data is about $7 \mathrm{~min}$. Table 1 summarizes the properties of the spectral lines observed at the VTT: $\lambda_{0}$ is the laboratory wavelength (taken from Nave et al. 1994 for Fe I) and $g_{\text {eff }}$ the effective Landé factor. The pixel size along the slit is given in the last column. The full Stokes vector is available for all visible and infrared lines. For Ca II H, only the intensity profile was recorded due to the low light level. Figure 1 displays maps of the observed area for the first scan after the coarse alignment described in Appendix B.2.

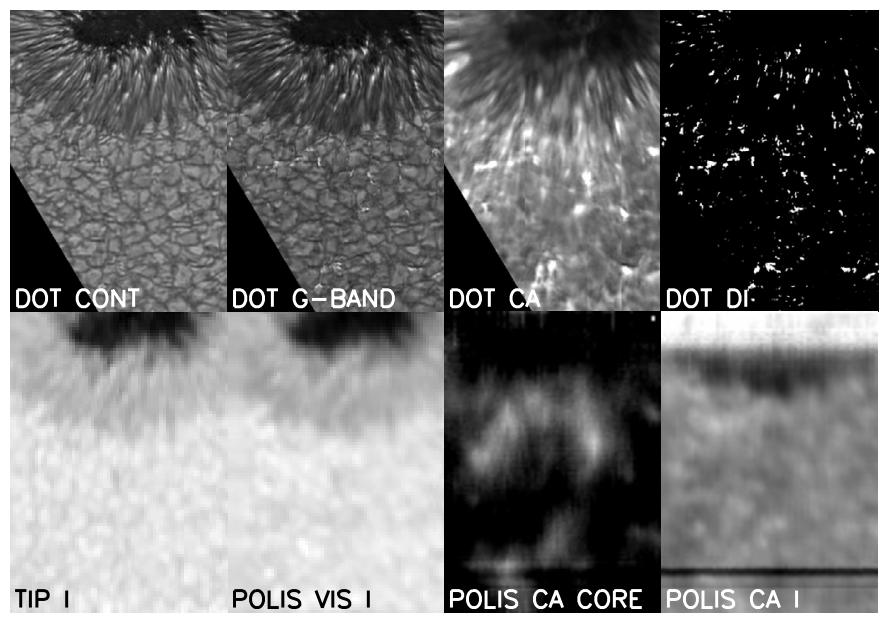

Fig. 1. Maps of the first scan across the limb-side part of NOAA 10425 after coarse alignment of DOT and VTT data. Top, left to right: blue continuum, G-band, and CaII H line-core filtergrams from the DOT, and difference between the G-band and blue continuum images. Bottom, left to right: continuum intensity at $1.5 \mu \mathrm{m}$, continuum intensity at $630.4 \mathrm{~nm}$, intensity of the $\mathrm{K}_{2 \mathrm{~V}}$ emission peak of the Ca II $\mathrm{H}$ line, and intensity in the Ca II H line wing; data from the VTT.

\subsection{Observational setup at the DOT}

The data from the DOT consist of speckle-reconstructed filtergrams in the $\mathrm{G}$ band at $430.5 \pm 0.5 \mathrm{~nm}, \mathrm{Ca}$ II H at $396.8 \pm$ $0.06 \mathrm{~nm}$, and blue continuum at $432.0 \pm 0.3 \mathrm{~nm}$, with a cadence of $1 \mathrm{~min}$. The size of the FOV is $77^{\prime \prime} \times 60^{\prime \prime}$ and the spatial sampling 0.'071 per pixel. More detailed information on the image processing and data reconstruction can be found in Sütterlin et al. (2004). The angular resolution of the filtergrams reaches the diffraction limit of the telescope $(0.2$ at $430.5 \mathrm{~nm}$, cf. Appendix A).

\subsection{Calibration of polarimetric data}

Besides the usual dark and flatfield corrections, the polarization measurements were corrected for instrumental polarization. This was done in two steps. First, TIP and POLIS have internal calibration units in front of the CT optics. Using them, the crosstalk among the Stokes parameters due to the optics behind the calibration units can be determined and removed (Beck et al. $2005 b)$. The remaining optical path consists of the telescope proper, i.e., the two coelostat mirrors, the entrance and exit windows of the evacuated tube, the main mirror, and one folding mirror. The instrumental polarization induced by these elements was corrected using the telescope model of Beck et al. (2005a). Residual crosstalk between the different Stokes parameters is estimated to be on the order of $10^{-3} I_{\mathrm{c}}$. The rms noise in the profiles is $4 \times 10^{-4} I_{\mathrm{c}}$ for the IR spectra and $10^{-3} I_{\mathrm{c}}$ for the visible spectra, as evaluated in continuum windows.

\subsection{Alignment of data sets}

For the investigation of the magnetic properties of G-band BPs, which are identified using the DOT data, it is crucial to have a good alignment of the data sets from the two telescopes. Additionally, the simultaneous inversion of infrared and visible spectral lines requires the polarization signals in both wavelength ranges to be cospatial and cotemporal. Appendix A explains in detail the procedures we followed to align the different 


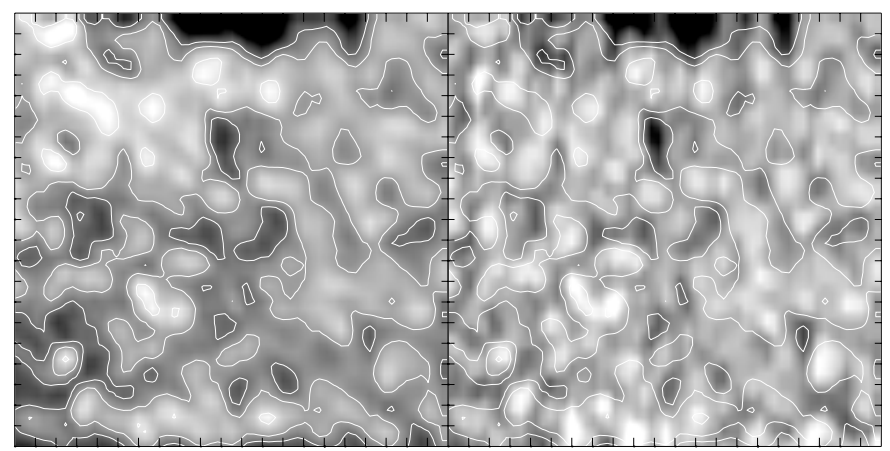

Fig. 2. Continuum intensity maps from POLIS (630 nm; left) and TIP (1565 nm; right). Only the lower part of the FOV is shown. Contours outline the brightest granules and darkest intergranular lanes observed in the POLIS map. Tick marks are separated by $1^{\prime \prime}$.

data sets. Figure 2 displays continuum intensity maps from TIP and POLIS after coalignment. As can be seen, the spatial correspondence of the structures in the two images is remarkably good. The accuracy of the alignment between TIP and the visible channel of POLIS is about 0.1 , or roughly a third of a TIP pixel. The maximum displacement perpendicular to the slit due to differential refraction (see, e.g., Reardon 2006) was 1", corresponding to a maximum time gap between cospatial Stokes profiles of 18 seconds ( 3 steps of 0 ! 35 ).

The time difference between cospatial VTT and DOT observations is $30 \mathrm{~s}$ or less. The spatial misalignment between VTT and DOT data is smaller than one TIP pixel, i.e., 0.35 . This is demonstrated in Fig. 3, where we display coaligned maps for the first scan across the FOV. Only the lower part of the FOV without the sunspot is shown. The intergranular lanes visible in the DOT G-band filtergram can clearly be traced in the IR continuum map. Appendix C contains all coaligned DOT and VTT maps.

\section{Data analysis}

The cospatial and cotemporal observations described in the previous section were used to investigate the magnetic, kinematic, and thermal properties of G-band BPs in the moat of NOAA 10425. As a first step, we set up a common wavelength scale for the visible and infrared lines. Next, a number of observables were extracted from the profiles. These line parameters, together with the results of the inversion of the observed spectral lines, allowed us to determine the physical properties of the various structures in the FOV. Part of our analysis is based on a statistical study that compares the properties of BPs and nonBPs (NBPs), the latter being points in the FOV that do not show enhanced G-band brightness (cf. Sect. 3.4). In this section we give details on the wavelength scale setup, the extraction of line parameters, and the inversion procedure. We also explain how BPs and NBPs were identified in the DOT filtergrams and the VTT maps.

\subsection{Wavelength scale}

The wavelength scale depends on two factors: the spectral dispersion and the rest wavelength of the lines, $\lambda_{0}^{i}$, where index $i$ cycles through the spectral lines observed (cf. Table 1$)$. The dispersion affects, e.g., the determination of the magnetic field strength from the separation of the Stokes $V$ peaks, or any velocity defined by the relative displacement to $\lambda_{0}^{i}$.
To set up a common wavelength scale for TIP and POLIS, we calculated a mean quiet Sun (QS) intensity profile in the lower half of the FOV. This was done for each of the eight scans and each spectral region (IR or visible) separately. We then set the rest wavelengths of Fe I $1564.8 \mathrm{~nm}$ and Fe I $630.15 \mathrm{~nm}$ such that the observed positions of the line cores in the mean QS profile were at the predicted convective blueshift values (cf. the last row of Table 4). Convective blueshifts were calculated using the field-free, two-component QS model of Borrero \& Bellot Rubio (2002). Next, the dispersions were adjusted until Fe I $1565.2 \mathrm{~nm}$ and Fe I $630.25 \mathrm{~nm}$ showed the correct convective blueshifts. This procedure yielded a dispersion of $29.65 \mathrm{~m}^{\circ}$ pixel $^{-1}$ for the IR lines and $14.86 \mathrm{~m}^{\circ}$ pixel $^{-1}$ for the visible lines. To remove the temporal evolution of the relative motions between the Sun and the observer, a separate calibration was used for each scan.

The measurement of Doppler shifts relative to the rest wavelength of the lines should be accurate to $\pm 100 \mathrm{~m} \mathrm{~s}^{-1}$, as this is the uncertainty in determining line-core positions. However, the assumed rest wavelengths (and hence the zero points of the velocity scales) are more uncertain because of three possible influences: a reduction of convective blueshifts due to the presence of magnetic fields, a reduction of convective blueshifts because of the off-center position of the spot, and a global offset due to the moat flow. The first two effects would shift all velocities towards higher redshifts, because we enforce the lines in the mean QS profile to have the convective blueshift values appropriate for disc center. However, we believe the error due to a possible reduction of convective blueshifts to be small. First, the granulation appears to be relatively undisturbed in the region where the average QS profiles were computed (the moat flow prevents the formation of large-scale plage fields close to the spot). Second, Balthasar (1985) showed that convective blueshifts outside disk center are reduced, but only by a small amount (depending on the line).

With respect to the moat flow, it is important to recall that this is a large-scale coherent phenomenon. Since the profiles used to compute the average QS profiles are affected by the moat flow, referring the velocities to the mean QS profile takes out the wavelength shift induced by the moat flow in the same way as all other relative motions between observer and target are removed. The only influence of the moat flow could then be differential moat velocities across the FOV. Assuming a horizontal moat flow of around $0.5 \mathrm{~km} \mathrm{~s}^{-1}$ (Balthasar et al. 1996; Rezaei et al. 2006), we find that the maximum error due to differential velocities cannot be larger than $0.25 \mathrm{~km} \mathrm{~s}^{-1}$ at the heliocentric angle of the observations. The accuracy of the velocity scale thus only depends on how appropriate the convective blueshift values predicted by the QS model are. As the main effects (off-center location, presence of fields) would reduce the blueshift values in the QS profiles, all velocities derived from our wavelength scale may only be biased to show larger blueshifts than actually present.

We note that, prior to the determination of the wavelength scale, the profiles from the visible channel of POLIS have been corrected for the curvature of the spectrum along the slit. The curvature is caused by the short focal length of the spectrograph and the large FOV along the slit. To avoid errors in the velocity determination due to this effect, the spectra have been shifted individually so as to have the teluric $\mathrm{O}_{2} 630.20 \mathrm{~nm}$ line (which does not show Doppler velocities) at the same position as in the QS profile from which the wavelength scale is determined (cf. Rezaei et al. 2006). 


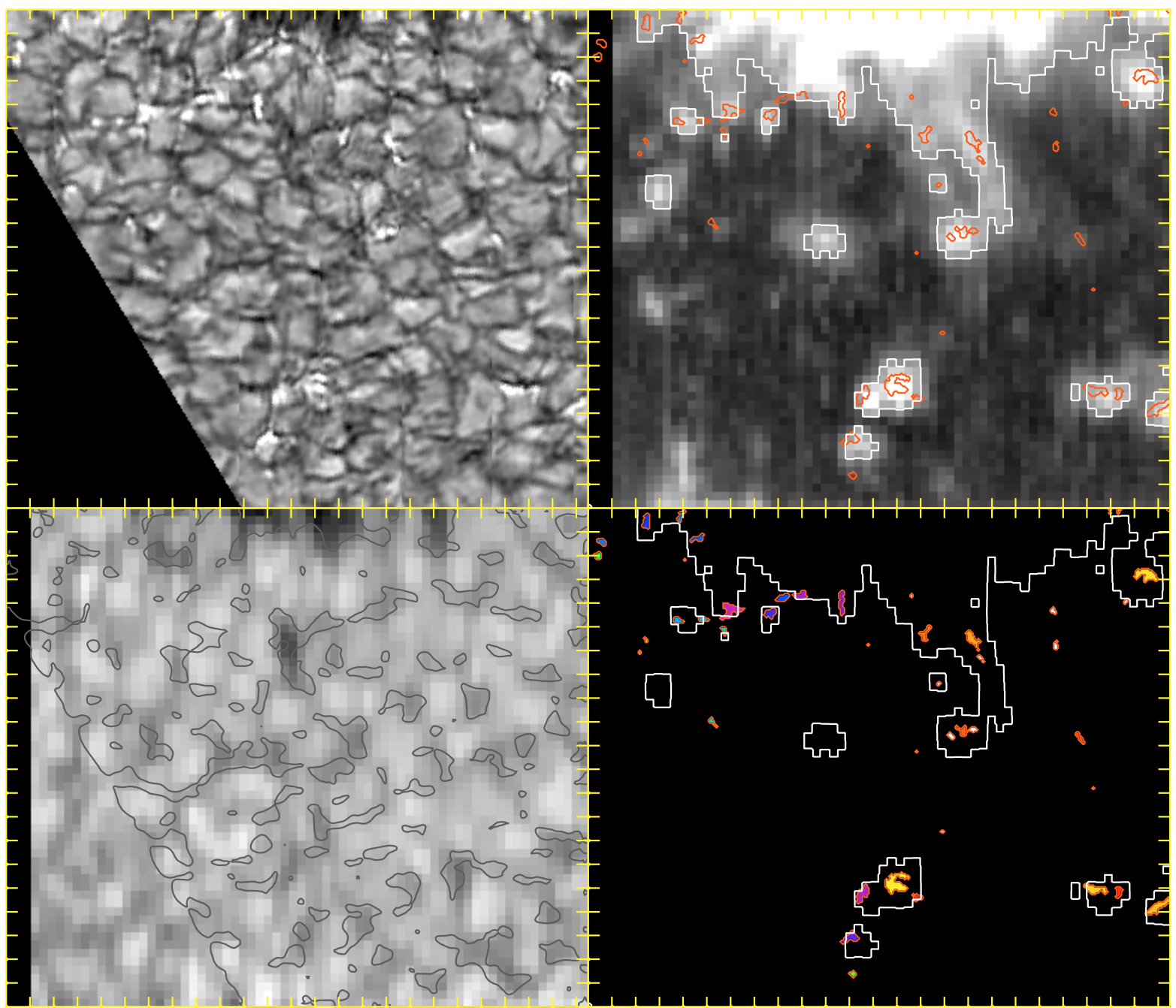

Fig. 3. Left panels: G-band intensity (top) and IR continuum intensity (bottom) for the first scan. The intergranular lanes visible in the G-band map can be traced in the IR intensity map as well (black contours) despite the inferior spatial resolution. Tick marks are separated by $1^{\prime \prime}$. Right panels: Total integrated polarization (top) and DOT BP mask (bottom). The individual BPs are color coded in the DOT BP mask. White contours outline strong polarization signals $(>1 \%)$ in the moat of the spot, as well as the sunspot canopy. Most of the identified BPs (red contours) coincide with areas of non-zero polarization signal (top right).

\subsection{Line parameters}

For each spatial point, we have the four Stokes profiles of the visible and IR lines listed in Table 1, the intensity profile of the Ca II $\mathrm{H}$ line from the POLIS UV channel, and the intensity in the $\mathrm{G}$ band, the $\mathrm{Ca}$ II $\mathrm{H}$ line core, and the blue continuum from the DOT, averaged over a $5 \times 5$ pixel area to simulate a TIP pixel. After alignment of the data sets, these observables are cospatial and cotemporal.

The four spectral lines with the highest magnetic sensitivity (Fe I $1564.8 \mathrm{~nm}$ and Fe I $1565.2 \mathrm{~nm}$ in the IR, Fe I $630.15 \mathrm{~nm}$ and Fe I $630.25 \mathrm{~nm}$ in the visible) have been used to extract the following line parameters:

- The line-core velocity from the intensity profiles (also for Ti I $630.37 \mathrm{~nm}$ ).

- The total integrated polarization, $\mathcal{T}$, defined as

$\mathcal{T}=\int\left(Q^{2}+U^{2}+V^{2}\right)^{1 / 2} / I_{\mathrm{c}} \mathrm{d} \lambda$,

in a wavelength range encompassing the full spectral line.

- The maximum polarization degree,

$p=\max \left\{\left(Q^{2}+U^{2}+V^{2}\right)^{1 / 2} / I\right\}$.
A profile is labeled unpolarized if the maximum polarization degree is below $0.2 \%$ (IR lines) or $0.5 \%$ (visible lines), otherwise it is called polarized. These thresholds correspond to 5 times the noise level. Pixels with at least one spectral line above the respective threshold were subject to a twocomponent inversion (cf. Sect. 3.3 below).

The following line parameters were calculated only for profiles showing polarization signal:

- The mean ratio of total linear and circular polarization, $L / V=\left\langle\left(Q^{2}+U^{2}\right)^{1 / 2} /|V|\right\rangle$, in a narrow wavelength range around the maximum absolute Stokes $V$ signal, i.e., the ratio at the position of the strongest $\sigma$ component.

- The area asymmetry of the Stokes $V$ profile,

$\delta A=s \times \frac{\int V(\lambda) \mathrm{d} \lambda}{\int|V(\lambda)| \mathrm{d} \lambda}$.

The polarity, $s$, is \pm 1 and denotes the orientation of the magnetic field vector to the LOS. It is derived from the position of minimum and maximum Stokes $V$ signal.

- The number and position (with pixel accuracy) of lobes in Stokes $Q, U$, and $V$. Lobes are defined as local extrema of 

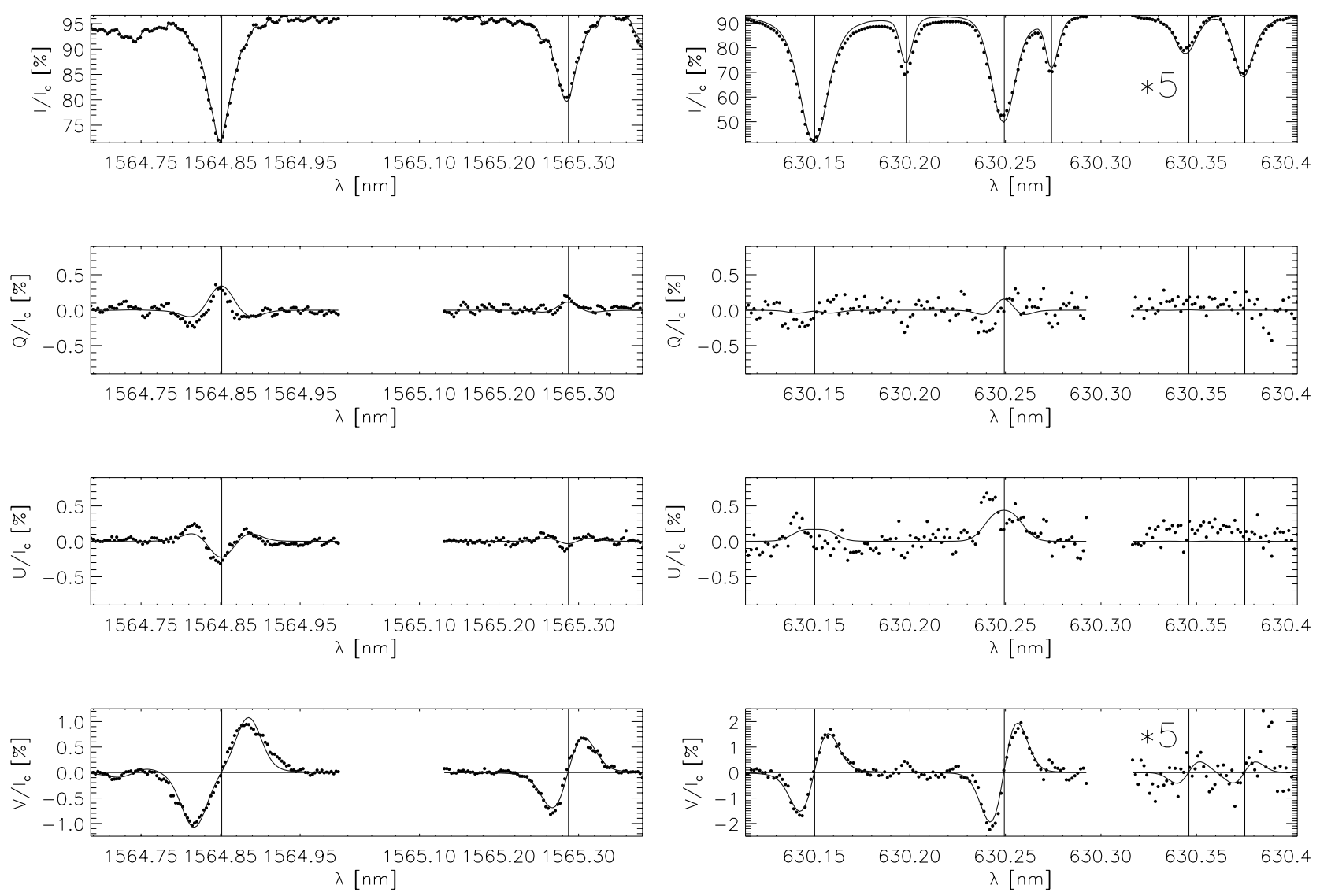

Fig. 4. Example of cospatial Stokes profiles from a G-band BP, normalized to the continuum intensity of the quiet Sun at disk center. From top to bottom: Stokes $I, Q, U$, and $V$. The vertical lines mark the rest wavelengths derived from the quiet Sun intensity profiles. The observed profiles are indicated by filled circles; the solid lines represent the best-fit profiles from the inversion. Left panel: Fe I line at $1564.8 \mathrm{~nm}$ with blend at $1564.7 \mathrm{~nm}$, and Fe I $1565.2 \mathrm{~nm}$. Right panel: Fe I lines at 630.15 and $630.25 \mathrm{~nm}$ with the teluric $\mathrm{O}_{2}$ lines at 630.200 and $630.276 \mathrm{~nm}, \mathrm{Fe} \mathrm{I} 630.35 \mathrm{~nm}$ and Ti I $630.37 \mathrm{~nm}$. The Stokes $I$ and $V$ profiles of the Ti I line are magnified by a factor of 5 for better visibility.

smoothed polarization profiles above a threshold of $0.1 \%$ for the IR lines and $0.25 \%$ for the visible lines.

Finally, for each Stokes $V$ profile with two distinct lobes, we calculated

- The Stokes $V$ amplitude asymmetry,

$\delta a=\frac{\left|a_{\text {blue }}\right|-\left|a_{\text {red }}\right|}{\left|a_{\text {blue }}\right|+\left|a_{\text {red }}\right|}$,

where $a_{\text {blue }}$ and $a_{\text {red }}$ represent the amplitudes of the blue and red lobes, respectively.

- The wavelength position of the $V$ lobes with sub-pixel accuracy for the derivation of field strengths.

- The Stokes $V$ zero-crossing velocity, $v_{\text {zcro }}$, from a linear fit to the circular polarization signal in between the lobes.

\subsection{Inversion procedure}

The four visible and the three infrared lines observed at the VTT were inverted together using the SIR code (Stokes Inversion based on Response functions; Ruiz Cobo \& del Toro Iniesta 1992). To facilitate the recovery of temperatures in deep photospheric layers, the Stokes profiles of each line were normalized to the continuum intensity of the quiet Sun at disk center. In addition, we removed the teluric blend of the Fe I $1564.8 \mathrm{~nm}$ line in the following way. The Fe I $1564.8 \mathrm{~nm}$ quiet Sun intensity profile was inverted with a two-component quiet Sun model. The bestfit profile does not contain the teluric blend, but reproduces the continuum outside the spectral line. The division of the observed with the best-fit profiles yields a multiplicative correction for the removal of the blend that was applied to all Fe I $1564.8 \mathrm{~nm}$ profiles.

The model atmosphere and corresponding free parameters depended on the type of pixel inverted (Table 2). The free parameters in the case of field-free atmospheres were the temperature, $T$, and line-of-sight (LOS) velocity, $v$. For magnetic atmospheres we also had the field strength, $B$, the LOS inclination, $\gamma$, and the azimuth of the field in the plane perpendicular to the LOS, $\psi$. The model atmosphere was given as a function of continuum optical depth, $\tau$, in the range from $\log \tau=1$ to -4 . The temperature was determined using two nodes, i.e., the absolute value and slope of the initial stratification could be modified. All other atmospheric parameters were assumed to be constant with depth. Our choice of height-independent magnetic and kinematic parameters has been made on purpose to facilitate the analysis of such a large data set. Model atmospheres with height-independent parameters cannot reproduce the asymmetries of the observed Stokes profiles, but have been shown to provide reasonable averages of atmospheric parameters along the LOS. This is exactly what is needed for a statistical analysis like the present one, where we focus on the general properties of G-band BPs rather than on the details of line formation. 
Table 2. Free parameters for the inversion of unpolarized pixels (second column) and polarized pixels (third and fourth columns). The meaning of the symbols is as follows: $\times$, free parameter; -, not used; 0 , same value in both atmospheric components.

\begin{tabular}{lccc}
\hline Parameter & Unpolarized & $\begin{array}{c}\text { Polarized } \\
\text { 1st comp }\end{array}$ & $\begin{array}{c}\text { Polarized } \\
\text { 2nd comp }\end{array}$ \\
\hline Temperature $(T)$ & $\times$ & $\times$ & $\times$ \\
Velocity $(v)$ & $\times$ & $\times$ & $\times$ \\
Field strength $(B)$ & - & - & $\times$ \\
Field inclination $(\gamma)$ & - & - & $\times$ \\
Field azimuth $(\psi)$ & - & - & $\times$ \\
Microturbulence $\left(v_{\text {mic }}\right)$ & $\times$ & $\times$ & $\times$ \\
Macroturbulence $\left(v_{\text {mac }}\right)$ & $\times$ & 0 & 0 \\
Straylight factor $(\beta)$ & - & 0 & 0 \\
Filling factor $(f)$ & - & 0 & 0 \\
\hline
\end{tabular}

The synthetic profiles were convolved with a macroturbulent velocity $v_{\mathrm{mac}}$. In our case, $v_{\mathrm{mac}}$ describes the effects of largescale motions and the unknown point spread functions (PSFs) of the spectrographs. This treatment, however, is simplistic because TIP and POLIS have slightly different PSFs. The microturbulence, $v_{\text {mic }}$, was another free parameter of the inversion. The code determines $v_{\text {mac }}$ and $v_{\text {mic }}$ separately for each inverted pixel. However, when two-component model atmospheres were used, the value of $v_{\text {mac }}$ was forced to be the same in both components. We employed one-component, field-free model atmospheres to invert pixels with no polarized profiles. The initial temperature stratification was that of the Harvard Smithsonian Reference Atmosphere (HSRA, Gingerich et al. 1971). No stray light was allowed for. The free parameters of the inversion were $T, v, v_{\text {mac }}$, and $v_{\text {mic }}$.

Pixels that were classified as polarized (cf. Sect. 3.2) were inverted with a two-component model. The first component was identical to the field-free atmosphere described above. The second component was magnetic. The Stokes profiles emerging from the two components, $p_{\text {mag }}$ and $p_{\text {nmag }}$, are combined to yield

$p_{\text {model }}(\lambda)=(1-f) p_{\text {nmag }}(\lambda)+f p_{\text {mag }}(\lambda)$,

where $f$ represents the magnetic filling factor, i.e., the fractional area of the pixel occupied by the magnetic component. Additionally, some amount of stray light contamination was assumed. We adopted the quiet Sun intensity profile as an approximation to the stray light profile. In this way, the synthetic profile used to fit the observations is

$p(\lambda)=\beta p_{\text {stray }}(\lambda)+(1-\beta) p_{\text {model }}(\lambda)$,

where $\beta$ represents the stray light factor. This combination is intended to describe Stokes profiles emerging from unresolved flux concentrations and their field-free surroundings, affected by stray light coming from unresolved granules and intergranular lanes. It is important to point out that $\beta$ and $f$ have different effects on the position and shape of the intensity profiles, which makes it possible to distinguish between them. The stray light profile corresponds to an additional field-free atmospheric component with fixed velocity and fixed temperature stratification. In contrast, the field-free component of the inversion can be adjusted both in velocity and temperature to represent the physical conditions of the plasma in the immediate vicinity of the flux concentration. We have chosen the simplest model possible for the inversion in order to have a robust estimate of magnetic properties of G-band BPs, which of course has some drawbacks. For example, the restriction to parameters that are constant in optical depth does not allow to reproduce asymmetries in the Stokes profiles.

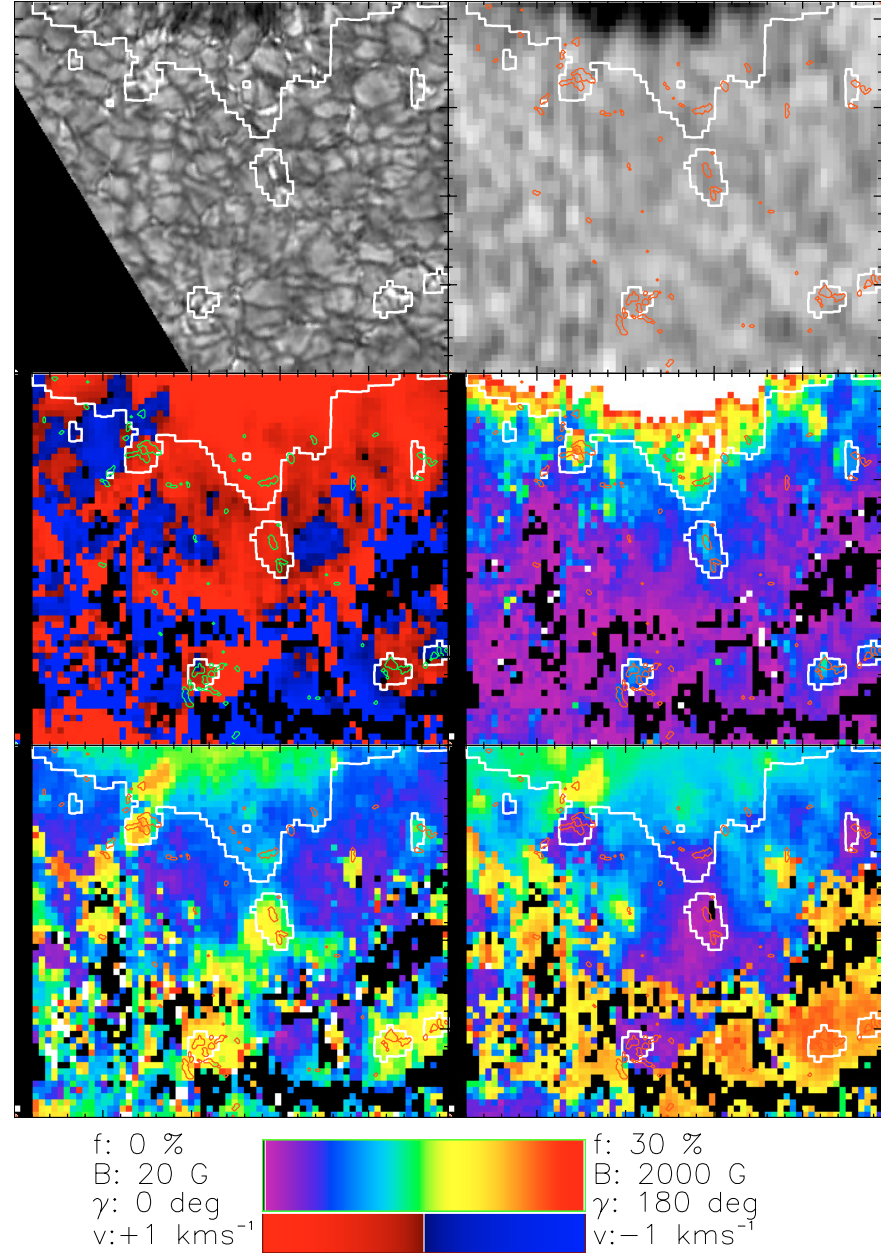

Fig. 5. Atmospheric parameters deduced from the inversion of the seventh map. Top: G-band intensity and IR continuum intensity. Middle: LOS velocity of the magnetic component and magnetic filling factor. Bottom: field strength and field inclination to the LOS. White contours outline areas of enhanced polarization signal and red contours (green in the velocity map) the identified BPs. Tickmarks are arcsec. Black areas represent pixels without polarization signal that have not been inverted in terms of a two-component model.

The uncertainties in the atmospheric parameters resulting from the inversion were estimated through the diagonal elements of the covariance matrix (Press et al. 1986). Averaged over the 4000 inverted profiles of the first scan, the formal errors in $B$, $\gamma, v$, and $f$ turned out to be $100 \mathrm{G}, 10^{\circ}, 400 \mathrm{~m} \mathrm{~s}^{-1}$, and $2 \%$. A number of factors may contribute to the relatively large error in velocity: possible height-variations of the flows that cannot be accounted for by our simple model, errors in the convective blueshift values $\left( \pm 150 \mathrm{~m} \mathrm{~s}^{-1}\right.$ for each line), and uncertainties in the laboratory wavelengths (especially for Ti I $630.37 \mathrm{~nm}$ ). Yet, these errors are smaller than the uncertainties associated with magnetogram or Dopplergram observations. The reason is the large amount of information we have at our disposal: the four Stokes profiles of seven different lines, which are used to constrain the model parameters simultaneously. Examples of observed and best-fit profiles are displayed in Fig. 4. Maps of the atmospheric parameters resulting from the inversion are shown in Fig. 5. 


\subsection{Identification of $B P s$}

In order to identify BPs in the DOT data, we calculated the relative $G$-band enhancement or $G$-band contrast map, $C$, using the G-band intensities, $I_{\mathrm{GB}}$, and blue continuum intensities, $I_{\mathrm{BC}}$, as follows:

$C=\frac{I_{\mathrm{GB}} \cdot\left\langle I_{\mathrm{BC}}\right\rangle /\left\langle I_{\mathrm{GB}}\right\rangle-I_{\mathrm{BC}}}{\left\langle I_{\mathrm{BC}}\right\rangle}$,

where $\langle I\rangle$ denotes the spatial average of the intensity over the map. Only strong enhancements of the G-band intensity relative to the blue continuum intensity are left over. An example can be found in Fig. 1.

The BPs proper were taken to be all closed contours in the G-band contrast map above a threshold of 0.31 . This value was selected by trial and error so as to encompass all BPs an observer would have identified visually in the G-band images of the first scan. Our procedure misses G-band BPs with low contrasts, but the detection of these BPs is always problematic independent of the method used to identify them. Contours near the upper edge of the FOV were not considered in the analysis to avoid contamination by the sunspot canopy. A few very short contour lines extending over 1-5 DOT pixels were excluded manually since they are presumably due to spurious signals created by the alignment procedure. Contours tracing G-band brightenings inside or at the edge of granules were removed as well. No further criterion on the contrast or the shape of the areas was applied. The closed contours were consecutively numbered to address individual BPs. In total, 447 separate BPs were identified in the full data set. Our selection of bright areas by sharp thresholding leads to extended patches, which would perhaps be split into smaller patches by the use of more sophisticated methods like the one proposed by Bovelet \& Wiehr (2003).

A BP mask for the polarimetric data from the VTT was constructed by marking all VTT pixels cospatial to one or more pixels above the contrast threshold in the DOT data. The 447 BPs identified in the G-band contrast maps extend over 1238 VTT pixels. The control sample of non-bright points (NBPs) was taken to be all VTT pixels at least three pixels (1'.05) apart from any BP and outside the canopy of the spot (visible near the upper edge of the FOV). An example of the NPB mask is displayed in Fig. C.1 for the first map. In total, around 9500 pixels were selected for the NBP sample.

The majority of BPs identified by the DOT mask have sizes below the spatial resolution of the polarimetric data. Part of the polarized light produced by these unresolved sources will therefore be distributed over several VTT pixels according to the spatial point spread function. The construction of the mask for the VTT data thus corresponds to choosing the spectra of the location where the signature of the BP should be maximum. However, light from other polarization sources inside the resolution element not related to a specific BP can also contaminate the spectra attributed to the BP.

\subsection{Data representation}

In the next sections, the parameters we use to describe the individual BPs are averaged over the spatial extension of each BP in the DOT mask and, analogously, over the corresponding area in the VTT mask if the BP extends over more than one pixel. The NBPs always encompass single pixels, so no averaging is carried out.

Individual G-band BPs may show different physical properties (e.g., magnetic flux, field strength, LOS velocity); in
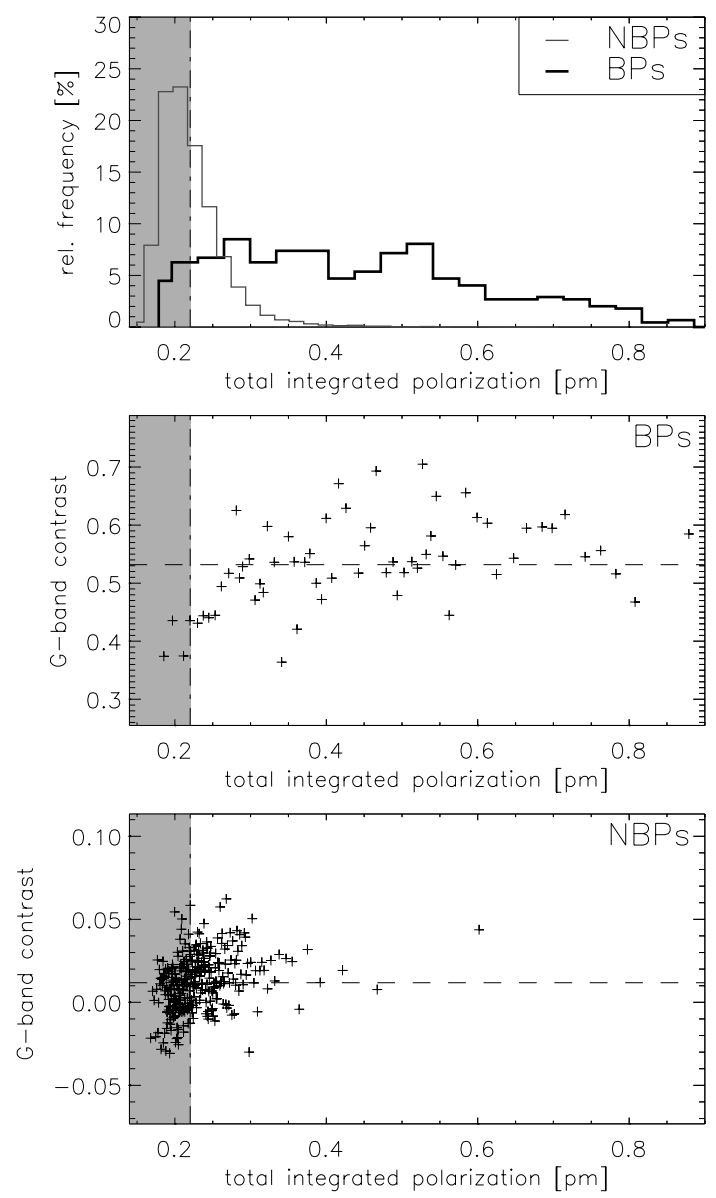

Fig. 6. Top: relative frequency of total integrated polarization values for BPs (thick black) and NBPs (thin grey). Middle and bottom: G-band contrast vs total integrated polarization for BPs and NBPs. The dashed horizontal lines indicate the mean contrast of 0.52 for BPs and 0.01 for NBPs. The shaded areas denote the range of $\mathcal{T}$ values within the noise level.

addition, there is noise in the data. Both factors lead to an unavoidable scatter that hides possible relations between the parameters characterizing the BPs. In an attempt to reveal these relations more clearly, we have chosen to investigate mean parameters obtained by binning the values of individual BPs. For all scatter plots relating two quantities, e.g., field strength and G-band contrast, we sorted the pairs $(x, y)$ of abscissa, $x$, and ordinate, $y$, to be in ascending order in $x$. We then averaged a variable number, $N$, of values in $x$ (typically $N=5-50$ ), and averaged the values of the dependent variable $y$ over the same pairs. With this procedure we keep the same statistics for each point plotted, as it is always derived from the same number of $(x, y)$-pairs. The range in $x$ over which the averaging is performed depends on how dense the values in $x$ are distributed after sorting.

Thus, each data point represents an average, and the error bar indicates the uncertainty of the mean, $\sigma / \sqrt{N}$, where $\sigma$ is the standard deviation of the averaged values in $y$. The implicit assumption here is that for a trend of, e.g., G-band contrast with field strength, other quantities like field inclination are randomly distributed, and their influence cancels out by the averaging process such that only the dependence on field strength is left over. Whenever possible, the noise level is estimated from the sample of NBPs. In most cases, the corresponding results for the 
Table 3. Fraction of unpolarized and polarized profiles of different types associated with BPs and NBPs, in percent.

\begin{tabular}{|c|c|c|c|c|c|c|}
\hline \multicolumn{7}{|c|}{ BP profiles, 1238 in total } \\
\hline \multirow{2}{*}{$\begin{array}{l}\text { Line } \\
\text { (nm) }\end{array}$} & \multirow[t]{2}{*}{ Unpolarized } & \multicolumn{5}{|c|}{ Polarized } \\
\hline & & Total & Regular & Irregular & Blue only & Red only \\
\hline $\begin{array}{l}1564.8 \\
\end{array}$ & 3 & 97 & (35) & (61) & $<1$ & $<1$ \\
\hline 1565.2 & 11 & 89 & 70 & 16 & 3 & $<1$ \\
\hline 630.15 & 12 & 88 & 80 & 7 & $<1$ & 1 \\
\hline 630.25 & 5 & 95 & 83 & 6 & 3 & 3 \\
\hline \multicolumn{7}{|c|}{ NBP profiles, 9497 in total } \\
\hline Line & Unpolarized & & & Polari & zed & \\
\hline$(\mathrm{nm})$ & & Total & Regular & Irregular & Blue only & Red only \\
\hline 1564.8 & 33 & 67 & (33) & $(26)$ & 6 & 2 \\
\hline 1565.2 & 72 & 28 & 15 & 4 & 7 & 2 \\
\hline 630.15 & 74 & 26 & 20 & $<1$ & 3 & 2 \\
\hline 630.25 & 49 & 51 & 22 & 2 & 20 & 7 \\
\hline
\end{tabular}

NPBs are also shown for comparison. The statistics of the NPBs are better due to the larger number of data points.

\section{Results}

Our results derive either directly from the spectral line profiles or from the more complex inversion procedure. In some cases, we used both approaches to confirm specific findings, while in other cases they were complementary.

\subsection{Polarization signature of $B P s$}

A visual inspection of Figs. 3, 5, or C.1 suggests that the majority of G-band BPs are associated with polarization signals. To quantify this impression, we use the total integrated polarization, $\mathcal{T} . \mathcal{T}$ is calculated separately for the four most Zeemansensitive spectral lines according to Eq. (1), and then averaged over all lines. We estimate the noise level in $\mathcal{T}$ by averaging $\mathcal{T}$ over all profiles that were not inverted due to their maximum polarization degrees being below the corresponding threshold. The average value plus one standard deviation, $\mathcal{T}_{\text {mean }}+\sigma$, is $0.22 \mathrm{pm}$ for these profiles. Values below this limit are assumed to correspond to the integration of pure noise, where of course we cannot exclude the existence of weak polarization signals below the noise level. The histogram of $\mathcal{T}$ values (upper panel of Fig. 6) demonstrates that the majority of BPs (94\%) have polarization signals above this level, whereas half of the NBPs have $\mathcal{T}$ values below it. Thus, clear polarization signals (i.e., magnetic fields) are observed no farther than 0.'35 from the position of almost all G-band brightenings. Due to the $1^{\prime \prime}$ spatial resolution of the spectra we cannot exclude that the polarization signal comes from a source outside the pixel itself, but we think it highly improbable that it happens so often by chance. The middle and lower panels of Fig. 6 display the G-band contrast of BPs and NBPs as a function of $\mathcal{T}$. We find that the contrast of BPs only depends on $\mathcal{T}$ for small polarization signals on the order of the integrated noise. For $\mathcal{T}$ values higher than $0.3 \mathrm{pm}$, the BP G-band contrast is approximately constant. The G-band contrast stays close to zero for the NBPs and shows no systematic trend with $\mathcal{T}$. This suggests that the NBP sample is distinctly different from the BP sample, even if it contains locations that exhibit some polarization signal.

The shape of the Stokes $V$ profiles gives information about the geometry of the magnetic field and the dynamical state of the flux concentrations. After the distinction between polarized and unpolarized profiles, the polarized profiles (cf. Sect. 3.2) were

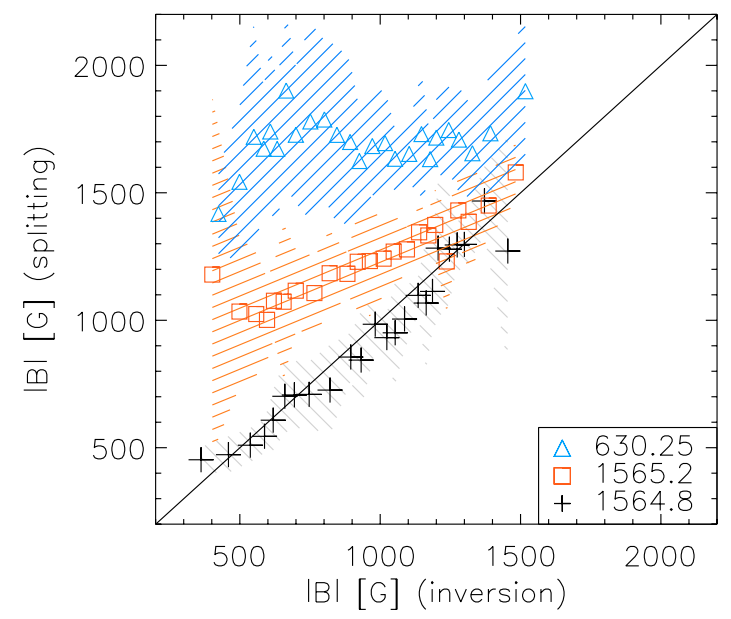

Fig. 7. Magnetic field strength of BPs from the Zeeman splitting of Fe I $1564.8 \mathrm{~nm}(+), 1565.2 \mathrm{~nm}(\square)$, and $630.25 \mathrm{~nm}(\triangle)$, vs. the values inferred from the inversion. The shaded areas give the scatter inside the bins. The straight line indicates a one-to-one correspondence.

classified according to the number of Stokes $V$ lobes. A profile is called regular if it has 2 lobes, irregular if it shows 3 or more lobes, and blue/red only if it shows only one blue/red lobe at shorter/longer wavelength than the intensity minimum. The Fe I line at $1564.8 \mathrm{~nm}$ is blended with Fe I $1564.7 \mathrm{~nm}$, which explains the high number of irregular profiles; the respective numbers are less reliable and thus are given in parentheses (Table 3). We find that roughly $80 \%$ of the BP profiles are regular with two lobes in Stokes $V$, while $10 \%$ are irregular with either one or three lobes, or unpolarized (10\%). In the sample of NBPs, the fraction of unpolarized and irregular Stokes $V$ profiles is significantly larger. A high percentage of BPs are associated with regular two-lobed Stokes $V$ profiles. There are few cases of irregular three-lobed profiles, which would indicate fields with opposite polarity and different LOS velocities in the same pixel. Examples of this kind can be found in the sunspot penumbra at the upper boundary of the FOV. Thus, even if the asymmetries of the Stokes $V$ profiles (cf. Sect. 4.5) indicate some variation in the magnetic properties along the LOS, most BPs do not seem to be related to a complex multi-component field topology.

\subsection{Magnetic field strength}

For spectral lines in the strong field limit, where the separation of the $\sigma$ components is larger than the thermal Doppler broadening, the magnetic field strength $(B)$ can be calculated from the position of the Stokes $V$ lobes according to

$\Delta \lambda=4.67 \times 10^{-13} g_{\mathrm{eff}} B \lambda_{0}^{2}$,

where $\Delta \lambda$ is the wavelength distance between the peaks of Stokes $V$ and the line center. In observations of magnetic fields outside sunspots or pores, Eq. (8) only fully applies to the IR line at $1564.8 \mathrm{~nm}$ for fields above $\sim 500 \mathrm{G}$. However, for a comparison with the inversion results, we calculate $B$ from Eq. (8) for the three most Zeeman-sensitive lines.

Figure 7 displays the BP field strengths indicated by the splitting of the different lines versus the field strengths inferred from the inversion. Taking the latter as the most accurate ones, it is clear that the splitting of Fe I $1564.8 \mathrm{~nm}(+)$ serves as a good diagnostics of $B$ for fields down to $500 \mathrm{G}$. The Fe I $1565.2 \mathrm{~nm}$ line $(\square)$, and the visible line at $630.25 \mathrm{~nm}(\triangle)$ especially, show 

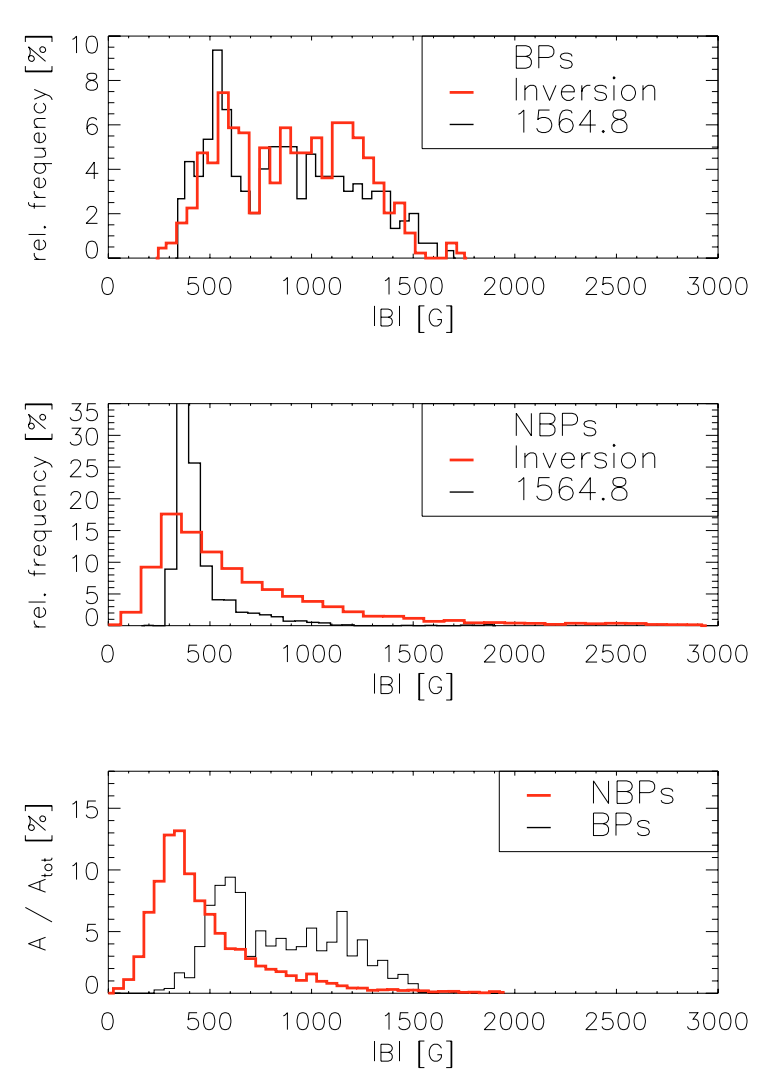

Fig. 8. Top: distribution of BP field strengths as inferred from the inversion (thick line) and the splitting of the Fe I $1564.8 \mathrm{~nm}$ line (thin line). Middle: same for the NBP sample. Bottom: fraction of the total area occupied by fields of given strength.

strong deviations for fields below $1200 \mathrm{G}$. The same result was obtained for the NBP sample.

The distribution of field strengths is displayed in Fig. 8 for individual BPs and NBPs. The inversion and the splitting of Fe I $1564.8 \mathrm{~nm}$ essentially give the same result: the field strengths of BPs are distributed uniformly in the range from $500 \mathrm{G}$ to about 1300-1400 G. The finding of G-band BPs with strengths of $\sim 500 \mathrm{G}$ may pose a serious problem for our understanding of why these flux concentrations are bright. In general, models predict zero or even negative G-band contrast for 500 G (Sánchez Almeida et al. 2001a; Shelyag et al. 2004), although high positive contrasts are also detected. The distribution of field strengths in the NBP control sample hints at a quickly decreasing probability for stronger fields. The same result has been found by Khomenko et al. (2003) in their study of quiet Sun internetwork fields using IR lines. The sharp drop of the relative frequency of fields weaker than $300 \mathrm{G}$ may be imposed by the detection limit of the observations (cf. Khomenko et al. 2003). Remarkably, it seems that the spot does not influence the distribution of weak fields in the outer part of the moat and surrounding quiet Sun much (the regions where the NBP sample comes from, cf. Fig. C.1). To allow comparisons with magnetoconvection simulations, the lower panel of Fig. 8 shows the area occupied by magnetic fields of a given strength using $100 \mathrm{G}$ bins, as a fraction of the total area occupied by fields in the BP and NBP sample, respectively. Figure 9 demonstrates that there is a slight increase in the G-band contrast toward higher field strengths for the BPs.

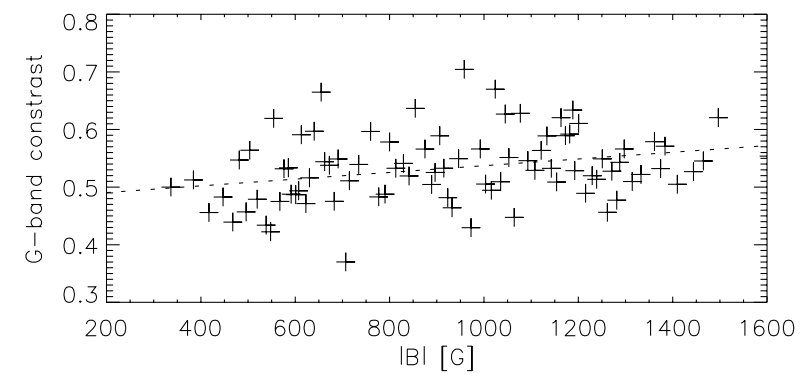

Fig. 9. G-band contrast vs field strength for the BP sample. The dashed line represents a linear fit to the data points.

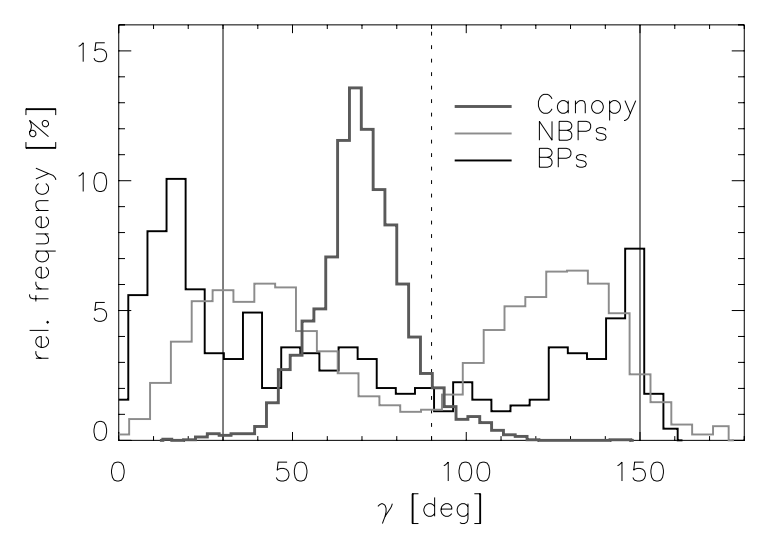

Fig. 10. Histogram of the magnetic field inclination with respect to the LOS for BPs (black), NBPs (grey), and the canopy area close to the sunspot (light grey). The vertical dashed line corresponds to fields perpendicular to the LOS. The vertical solid lines mark the LOS inclinations of fields, which are purely vertical relative to the solar surface.

\subsection{Magnetic field inclinations}

Figure 10 displays histograms of the LOS inclination for BPs and NBPs. Positive and negative polarities are found in both types of structures. In the case of the NBPs, the inclinations are distributed rather symmetrically around two broad peaks at $\gamma=40^{\circ}$ and $130^{\circ}$, with few fields perpendicular to the line of sight. For comparison, we also show the histogram of LOS inclinations found at the upper border of the FOV, close to the penumbra and its filaments (cf. Fig. C.1). The fields in this area exhibit a single peak at around $70^{\circ}$, which corresponds to the unipolar horizontal fields of the sunspot canopy. The distribution of LOS inclinations for the BPs peaks at around $15^{\circ}$ and $145^{\circ}$; the peaks are less broad than those of the NBP sample. Few BPs show fields perpendicular to the LOS. The LOS inclinations of BPs and NBPs at a heliocentric angle of $\theta=27^{\circ}$ are consistent to the first order with fields that are close to vertical to the surface. The systematic displacements toward smaller inclination (BP peak at $15^{\circ}, \mathrm{NBP}$ at $130^{\circ}$ ) could be due either to the influence of the canopy fields of the sunspot or to unresolved mixed polarity fields (Lites 2002).

The inclination of the vector magnetic field to the LOS can also be estimated from the ratio of linear to circular polarization, $L / V$. The $L / V$ ratio is more affected by noise in the data than the inclination derived from the inversion, especially for small 

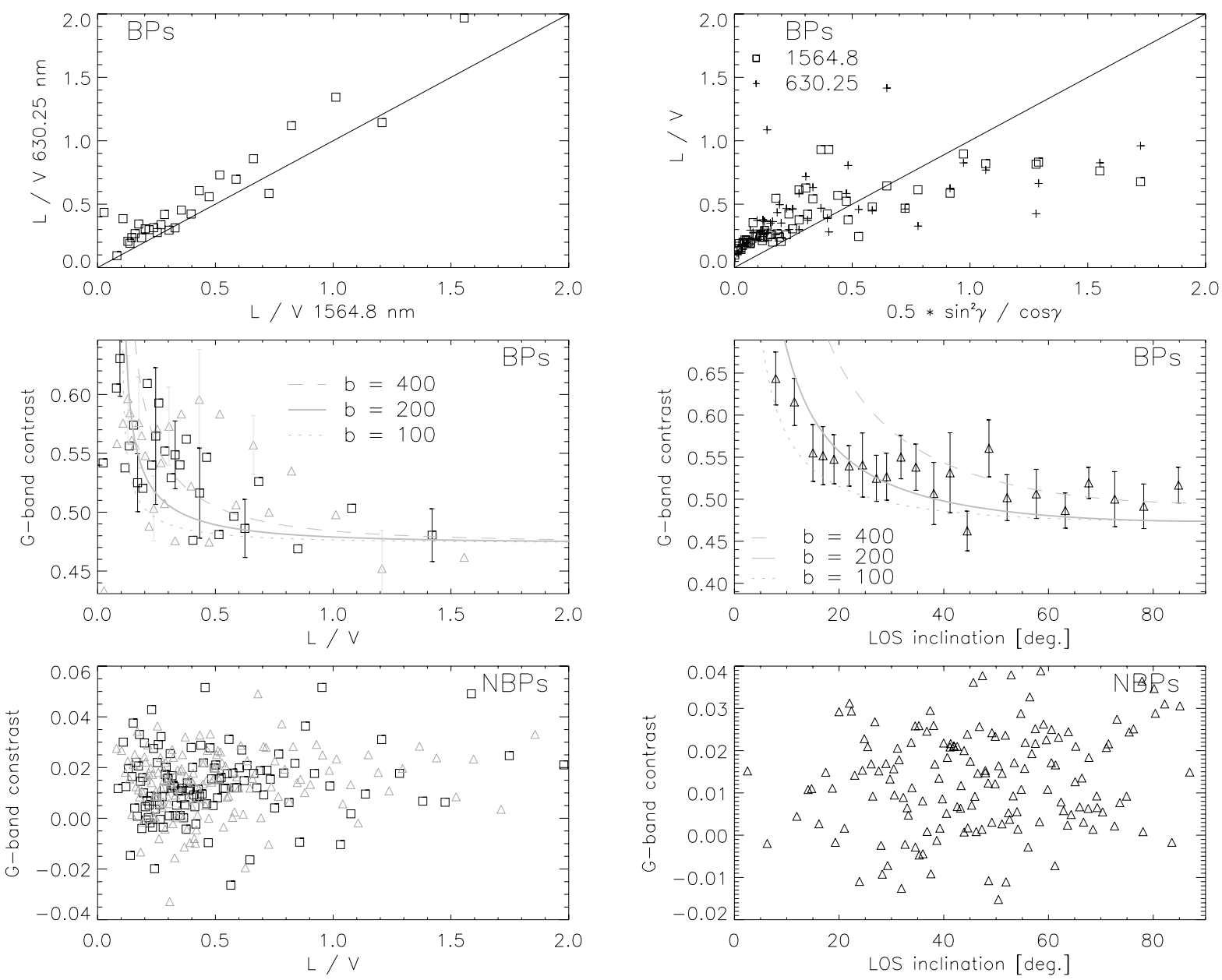

Fig. 11. Top panels: $L / V$ of Fe I $630.25 \mathrm{~nm}$ vs. the $L / V$ of Fe I $1564.8 \mathrm{~nm}($ left), and $L / V$ of $1564.8 \mathrm{~nm}(\square)$ and $630.25 \mathrm{~nm}(+)$ vs. the values obtained from Eq. (9) by inserting the inclinations deduced from the inversion (right). Middle left panel: dependence of the BP contrast on the $L / V$ ratio of $1564.8 \mathrm{~nm}(\triangle)$ and on the $L / V$ ratio averaged over the two visible lines $(\square)$. Middle right panel: dependence of the BP contrast on the field inclination deduced from the inversion. Overplotted are curves calculated from Eq. (11) for three values of the parameter $b$. Error bars indicate the uncertainty of the mean values. Bottom panels: BP contrast vs. L/V and vs. inclination, for NBPs.

amplitudes of $L$ or $V$. For fully split lines, Landi Degl'Innocenti (2003) demonstrate that

$$
L / V \simeq \frac{1}{2} \frac{\sin ^{2} \gamma}{\cos \gamma} \text {. }
$$

The upper left panel of Fig. 11 displays a scatter plot of the $L / V$ ratio for the two most Zeeman-sensitive spectral lines, Fe I $1564.8 \mathrm{~nm}$ and Fe I $630.25 \mathrm{~nm}$. The values of $L / V$ are similar in both wavelength regions; they only show significant deviations for values of $L / V$ above 0.45 or, according to Eq. (9), for inclinations larger than $50^{\circ}$. The upper right panel of Fig. 11 compares the $L / V$ values with the results of the inversion. This plot demonstrates that Eq. (9) is also valid for the visible Fe I $630.25 \mathrm{~nm}$ line if the field is not too much inclined $\left(\gamma \ll 50^{\circ}\right)$. In general, the observed values of $L / V$ are slightly higher than those indicated by the inversion, which we attribute to the influence of noise.

Interestingly, the G-band contrast of BPs seems to depend on the magnetic field inclination. The middle panels of Fig. 11 show the contrast as a function of both $L / V$ and the magnetic field inclination inferred from the inversion. We find a correlation of the brightest structures with magnetic fields parallel to the LOS. The BPs with magnetic fields perpendicular to the LOS systematically exhibit reduced G-band contrasts. We believe this trend to be significant even if the contrast shows some scatter due to intrinsic differences in the properties of the BPs like field strength or flux, which also have some effect on the contrast. The overplotted curves will be discussed in Sect. 5. No relation between the G-band contrast and the field inclination is observed for the NBPs (bottom panels of Fig. 11).

\subsection{LOS velocities}

We used two proxies to estimate flows of magnetized and fieldfree plasma: the Stokes $V$ zero-crossing shift and the Stokes $I$ line-core velocity, respectively. The inversion also yielded values for the LOS velocity in the magnetic and field-free components of the atmosphere. By convention, positive velocities indicate redshifts.

Figure 12 shows the dependence of the LOS velocity on the G-band intensity. For easier comparison with other data sets, we used the relative G-band intensity $1+C$ in this plot. For the NBP sample (small symbols in the left half of the figure, with relative intensities below 1.31), we find that the velocities derived from the Stokes $I$ line-core position and the velocities of the field-free component of the inversion are in good agreement with 


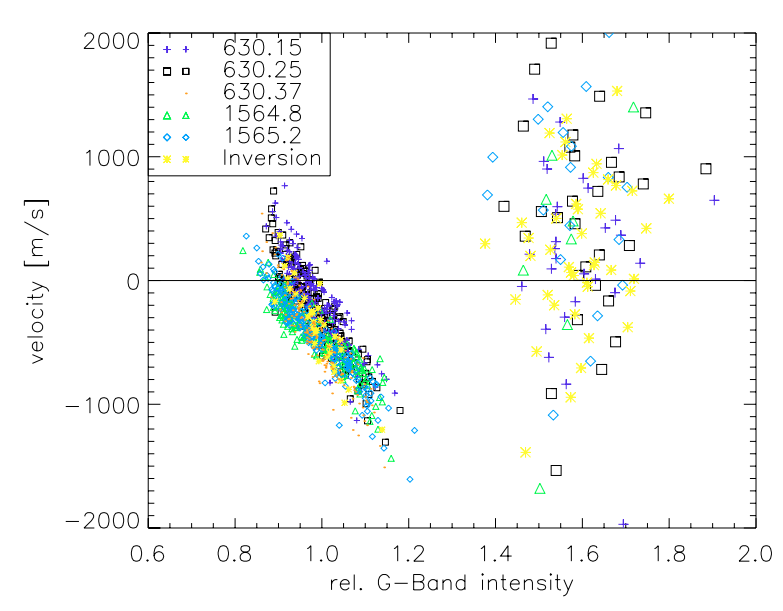

Fig. 12. Relation between the LOS velocity and the normalized G-band intensity. Positive velocities indicate redshifts. Small symbols: Stokes I line-core velocity and LOS velocity of the field-free component of the inversion for the NBP sample. Large symbols: Stokes $V$ zero-crossing velocity and LOS velocity of the magnetic component of the inversion for BPs.

Table 4. LOS velocities of BPs and NPBs in $\mathrm{m} \mathrm{s}^{-1}$.

\begin{tabular}{lccccc}
\hline \hline & 630.15 & 630.25 & 630.37 & 1564.8 & 1565.2 \\
\hline BPs, zero-crossing & 300 & 540 & & 394 & 598 \\
BPs, line core & -24 & -49 & -200 & -296 & -267 \\
NBPs, line core & -172 & -250 & -451 & -448 & -447 \\
Convective blueshift & -185 & -262 & -424 & -445 & -469 \\
\hline
\end{tabular}

the expectation for an area dominated by granulation: bright granules show blueshifts $(v<0)$, whereas intergranular lanes have reduced intensity and are associated with redshifts $(v>0)$.

In contrast, no trend of the magnetic velocity with intensity is found in the BPs (large symbols, relative intensities above 1.31 in Fig. 12). The velocity of the BPs spans the range $\pm 2 \mathrm{~km} \mathrm{~s}^{-1}$ for the binned data. The extreme velocities, considering only points with a clear polarization signal above $1 \%$, reach $\pm 4 \mathrm{~km} \mathrm{~s}^{-1}$. In general, all spectral lines and the inversion show a preference for downflows of magnetized plasma inside the BPs.

The magnetic velocity indicated by the inversion usually agrees with the Stokes $V$ zero-crossing velocity deduced from the visible lines (upper panel of Fig. 13), because a displacement between the observed and synthetic Stokes $V$ profiles would strongly degrade the quality of the fit, due to the steep slope of the circular polarization signal of the visible lines near the zero crossing. The IR line at $1565.2 \mathrm{~nm}$ usually shows the strongest redshifts. Fe I $1564.8 \mathrm{~nm}$ has less reliable statistics, as $v_{\text {zcro }}$ is only calculated for regular profiles (Table 3 ). The bottom panel of Fig. 13 compares the Stokes $V$ zero-crossing velocities derived from the IR and visible lines. The plot also shows a trend toward higher velocities in the IR as compared with the visible, as most points lie above the line of one-to-one correlation. This difference in magnetic velocity could be due to unresolved structures or flow fields, whose effect on infrared and visible lines may differ. It is also compatible with the existence of velocity gradients along the line of sight, as indicated by the profile asymmetries discussed in the next section.

Table 4 summarizes the average velocities of BPs and NBPs; positive values correspond to redshifts. The first and second row show the average zero-crossing velocity and the average
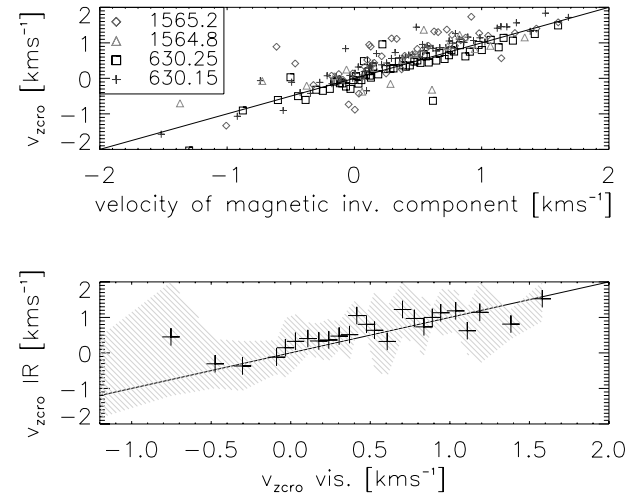

Fig. 13. Top: Stokes $V$ zero-crossing velocity, $v_{\text {zcro }}$, vs. the magnetic velocity from the inversion, for BPs. Bottom: BP zero-crossing velocities deduced from IR lines vs. those from visible lines. The zero-crossing velocity is averaged over the two visible (or infrared) lines when both show regular Stokes $V$ profiles. The shaded area indicates the scatter in the bins.

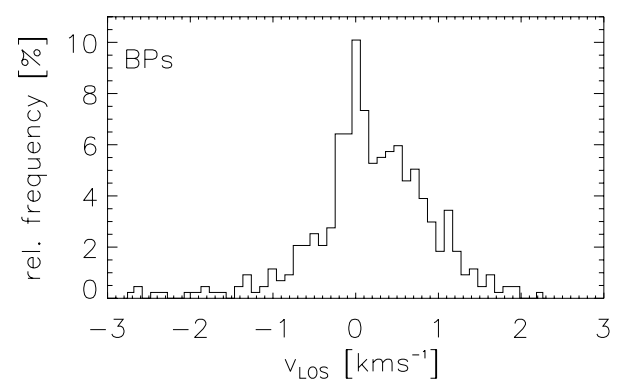

Fig. 14. Histogram of the LOS velocity of the magnetic component of the inversion, for BPs.

line-core velocity of BPs for the different spectral lines. The third row contains the average line-core velocity of NPBs. In the fourth row, the convective blueshifts from the two-component quiet Sun model of Borrero \& Bellot Rubio (2002) are given that were used to set up the wavelength scale. For BPs, the average zero-crossing velocities are between $300 \mathrm{~m} \mathrm{~s}^{-1}$ and $600 \mathrm{~m} \mathrm{~s}^{-1}$, depending on the spectral line; the average velocity of the magnetic component of the inversion is $260 \mathrm{~m} \mathrm{~s}^{-1}$ (cf. Fig. 14). These velocities agree with the results of Amer \& Kneer (1993), as far as different velocities are deduced from Stokes $I$ and Stokes $V$, and with Grossmann-Doerth et al. (1996) or Sigwarth et al. (1999), who found significant redshifts in magnetic elements. The line-core velocity of the BPs shows blueshifts. The magnetic elements and intergranular lanes are not resolved in the VTT data; thus, the intensity profiles of the BPs are probably affected by unpolarized light from bright granules in the immediate surroundings, which is a source of blueshifts. For the NBP sample, we find average velocities close to the convective blueshifts used in the determination of the wavelength scale, as expected. As discussed in Sect. 3.1, the velocities may be biased towards the blue.

\subsection{Area and amplitude asymmetries}

The area and amplitude asymmetries are sensitive to velocity and magnetic field gradients along the line of sight. Auer \& Heasley (1978) demonstrated that the existence of velocity gradients along the LOS is a necessary and sufficient condition for having non-zero area asymmetries (cf. also López Ariste 2002). Enhanced asymmetries result when gradients of velocity are 

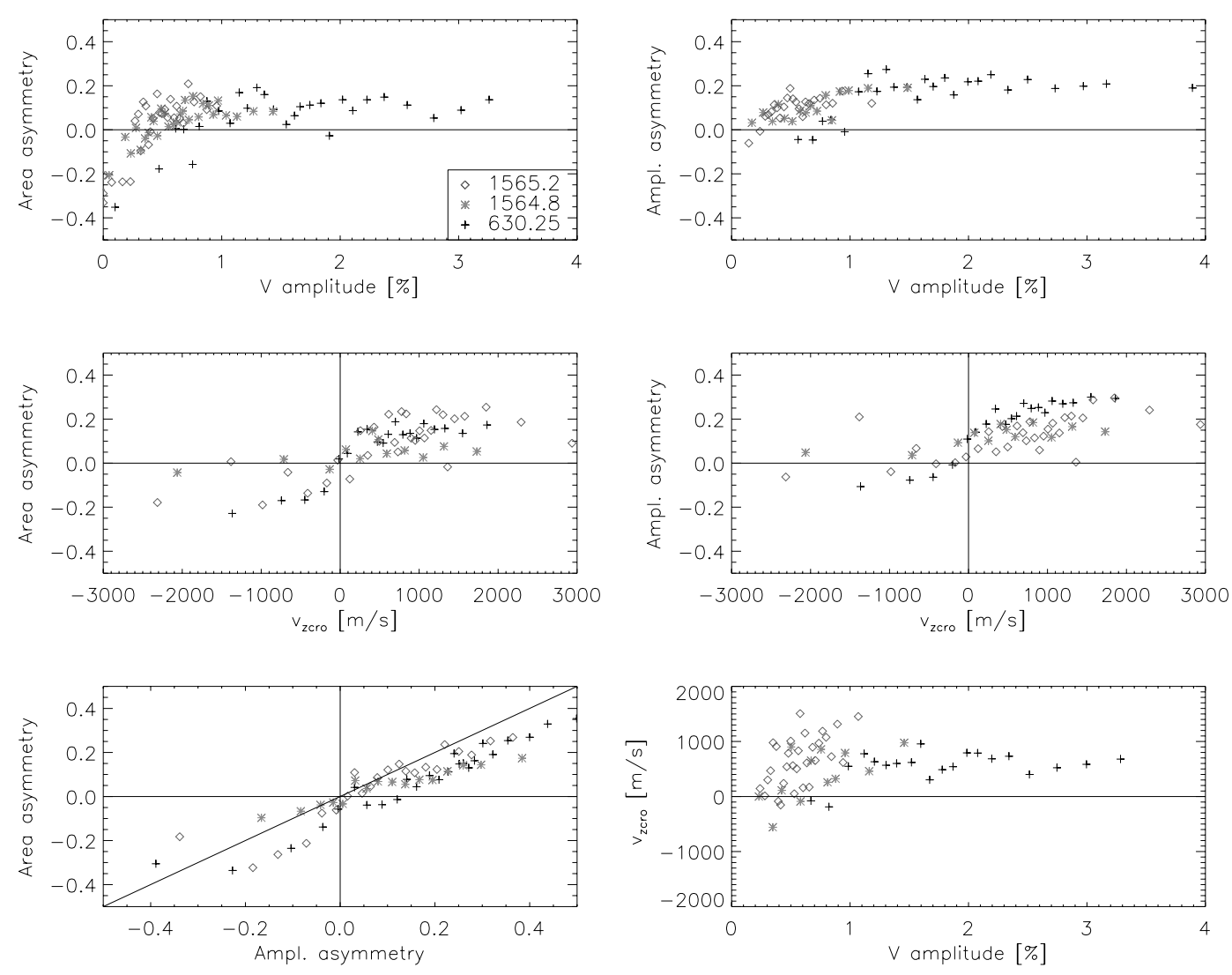

Fig. 15. Scatter plot of various Stokes $V$ line parameters for the BP sample. Left to right, top to bottom: $\delta A$ and $\delta a$ vs. Stokes $V$ amplitude, $\delta A$ and $\delta a$ vs. zero-crossing velocity, area asymmetry vs. amplitude asymmetry, and zero-crossing velocity vs. Stokes $V$ amplitude.

combined with gradients of magnetic field strength, field inclination, and/or field azimuth. Note that jumps of these atmospheric parameters at the interface between magnetic flux concentrations and their field-free surroundings would effectively produce gradients along the LOS. In the context of magnetic canopies, Solanki \& Pahlke (1988), Sanchez Almeida et al. (1989), or Sanchez Almeida (1998) have demonstrated that the sign of the area asymmetry is related to the sign of the gradients of velocity and field strength through

$\operatorname{sgn}(\delta A)=-\operatorname{sgn}\left(\frac{\mathrm{d} B(\tau)}{\mathrm{d} \tau} \frac{\mathrm{d} v(\tau)}{\mathrm{d} \tau}\right)$

In Fig. 15 we display the correlation between the asymmetries and other parameters for the BPs. The upper panels show that most BPs exhibit positive area and amplitude asymmetries, with values comparable to those found by Martínez Pillet et al. (1997) in plage regions and by Sigwarth et al. (1999) in network and internetwork areas. Negative asymmetries are encountered only for weak polarization signals. Both $\delta A$ and $\delta a$ change sign with the zero-crossing velocity (middle panels of Fig. 15). As a consequence, there are very few examples of, e.g., positive asymmetries associated with blueshifted profiles. The lower left panel of Fig. 15 shows that the correlation between area and amplitude asymmetry is quite tight for G-band BPs: structures with positive area asymmetry also have positive amplitude asymmetry, and when one increases the other follows it closely. Another finding is that the zero-crossing velocity of BPs depends on the amplitude of the polarization signal only for very low signals: most BPs with strong Stokes $V$ signals show similar velocities (cf. the lower right panel of Fig. 15).

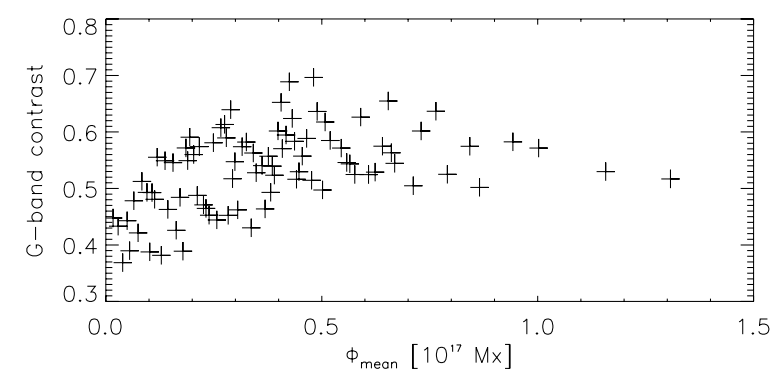

Fig. 16. G-band contrast vs. average single-pixel magnetic flux of BPs. The curve has a similar shape to the contrast vs. total integrated polarization (cf. Fig. 6).

\subsection{Magnetic flux}

The magnetic flux per pixel is calculated from the inversion results as $\Phi=(1-\beta) f A B \cos \gamma$, where $A=254^{2} \mathrm{~km}^{2}$ is the area corresponding to a VTT pixel. Figure 16 displays the variation of the G-band contrast of BPs with the average unsigned flux per pixel, $\langle\Phi\rangle_{\mathrm{BP}}$, where \langle\rangle$_{\mathrm{BP}}$ indicates the average over all VTT pixels covered by the BP. The contrast increases with magnetic flux until about $\langle\Phi\rangle_{\mathrm{BP}}=5 \times 10^{16} \mathrm{Mx}$ and then decreases. The relation between G-band contrast and mean unsigned flux is very similar to that between contrast and total integrated polarization $(\mathcal{T}$, cf. Fig. 6$)$. This supports the consistency of the inversion results: $\mathcal{T}$ is proportional to the flux and is derived directly from the observed profiles. The magnetic flux is calculated using three different free parameters of the inversion, but shows the same dependence with the G-band contrast. 

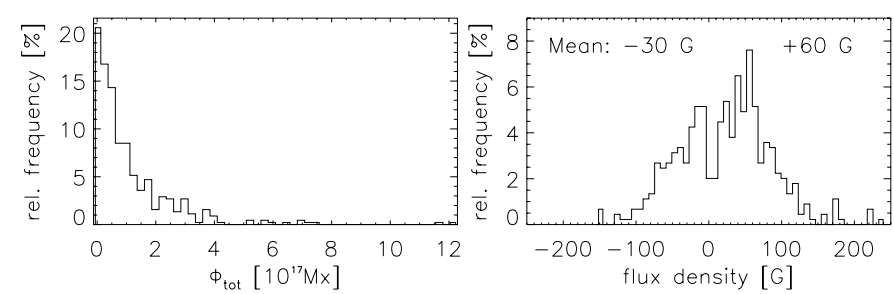

Fig. 17. Left: histogram of the total unsigned flux of BPs. Right: maximum flux density of BPs.

The total magnetic fluxes we infer for the BPs, $\Phi_{\text {tot }}=$ $n \cdot\langle\Phi\rangle_{\mathrm{BP}}$, with $n$ the number of VTT pixels covered by the BP, are similar to the ones obtained by Berger \& Title (2001), albeit generally smaller (see left panel of Fig. 17). For a comparison with their paper we also computed the maximum value of the flux density in each $\mathrm{BP}\left(\Phi_{\max }(\mathrm{BP}) / A\right.$; right panel of Fig. 17), for which we again find lower values than they do. The most probable value of $\Phi_{\max }(\mathrm{BP}) / A$ is around $+60 \mathrm{G}$ for positive-polarity BPs and $-30 \mathrm{G}$ for negative-polarity BPs, i.e., only about half the value reported by Berger \& Title (2001). This systematic difference may have two origins: (1) the different methods used to derive the magnetic flux, full vector polarimetry of several lines in the present study and magnetograms of a single line in the case of Berger \& Title (2001), which result in different noise levels and polarimetric sensitivities, and/or (2) the lower spatial resolution of our observations.

\subsection{Dependence of G-band contrast on temperature}

The temperature stratification of the BPs is derived from the inversion, both for the magnetic interior and the immediate fieldfree surroundings. To facilitate comparisons, we consider the mean temperature of each atmospheric component in the optical depth range from $\log \tau=0$ to $\log \tau=-2$. The relation between the G-band contrast and the temperature of BPs is displayed in the upper left panel of Fig. 18. The contrast shows no clear correlation with the temperature of either the magnetic interior or the field-free surroundings. However, the temperatures of the two components show systematic differences: the histograms displayed in the upper right panel reveal that the magnetic atmosphere is hotter than the immediate field-free surroundings by about $1000 \mathrm{~K}$ at equal optical depth. The mean temperature is $5800 \mathrm{~K}$ for the magnetic component and around $4800 \mathrm{~K}$ for the field-free surroundings.

\subsection{Dependence of the temperature on magnetic flux and field strength}

A slight reduction in the temperature of the magnetic interior with increasing magnetic flux is found, as can be seen in the lower left panel of Fig. 18. A similar correlation exists between the temperature of the magnetic component and the field strength (lower right panel of Fig. 18). The temperature of the field-free surroundings does not show any correlation with either the magnetic flux or field strength.

\subsection{The size of G-band BPs}

The quantity $f_{\text {mag }}=(1-\beta) f$ represents the fraction of a single VTT pixel occupied by magnetic fields, as estimated by the inversion code. It can be converted to the effective diameter $D$ of a circular structure with the same area. The upper limit of $D$ for a single pixel is $292 \mathrm{~km}$; however, some BPs cover $n$ VTT pixels. The size of each BP in the VTT maps is thus calculated from $n\left\langle f_{\text {mag }}\right\rangle_{\mathrm{BP}}$. In this derivation it is implicitly assumed that only a single source is generating the polarized light, even if the magnetic filling fraction only gives the area inside the pixel occupied by all fields. For the VTT, no lower size limit exists, because the magnetic filling factor can go smoothly towards zero. For the DOT images, the number of bright pixels belonging to a single BP structure is converted to an effective diameter. The minimum effective diameter for any BP in the DOT filtergrams is then $60 \mathrm{~km}$ ( $\equiv 1$ DOT pixel).

Figure 19 displays the histogram of BP diameters as inferred from both data sets under these assumptions. The maximum diameter is $500 \mathrm{~km}$ in the VTT maps and $700 \mathrm{~km}$ in the DOT filtergrams. We find a distribution with a mean value of $150 \mathrm{~km}$ for the VTT and $210 \mathrm{~km}$ for the DOT, with few BPs with diameters above $300 \mathrm{~km}$. However, the mean value may be misleading due to the skewness of the distribution. For comparison, the overplotted log-normal distribution would indicate $D \sim 90 \mathrm{~km}$ as the most probable BP diameter. In the DOT filtergrams, there is an increase of the relative frequency of small BP structures extending over 1 or 2 pixels $(60-100 \mathrm{~km})$. These structures are very likely artifacts introduced by the data processing and the alignment procedure of DOT and VTT data, where the DOT image closest in time is used without a smooth transition from one DOT image to the next. In principle, these smallest BPs should have been rejected as unphysical, because the DOT resolution of 0.2 forces the minimum diameter to be around $170 \mathrm{~km}$. Our manual removal of short contour lines thus seems to have been insufficient, but fortunately only a small fraction of the BPs is affected. Note that a more sophisticated algorithm for the identification of BPs would also most probably have resolved the larger structures into chains of smaller BPs. The BP sizes found here are comparable to the typical width of intergranular lanes and the BP sizes given by Berger \& Title (2001) and Wiehr et al. (2004), respectively.

The lower panel of Fig. 19 compares the effective diameters of BPs inferred from the VTT and the DOT observations. The BP sizes derived from the former are systematically smaller, amounting to only $78 \%$ of the DOT diameter on average. The smaller size may be related to the spatial resolution of the VTT spectra. The magnetic filling fraction is underestimated, as part of the polarized light is scattered out of the VTT pixel by seeing.

\section{Summary and discussion}

The analysis of cospatial and simultaneous G-band images and spectropolarimetric measurements reveals different aspects of both the statistics of BP fields and the relation between G-band intensities and magnetic properties of the flux concentrations. We find the following properties from a statistical analysis of 447 identified BPs:

- $94 \%$ of the BPs are cospatial with polarization signals above the noise level.

- The magnetic field strengths of BPs range from 500 to $1400 \mathrm{G}$. The field strength distribution is rather flat within this range.

- The total magnetic flux of BPs ranges from 0 to up to $4 \times$ $10^{17} \mathrm{Mx}$, with an average flux density per pixel of around $50 \mathrm{G}$. 

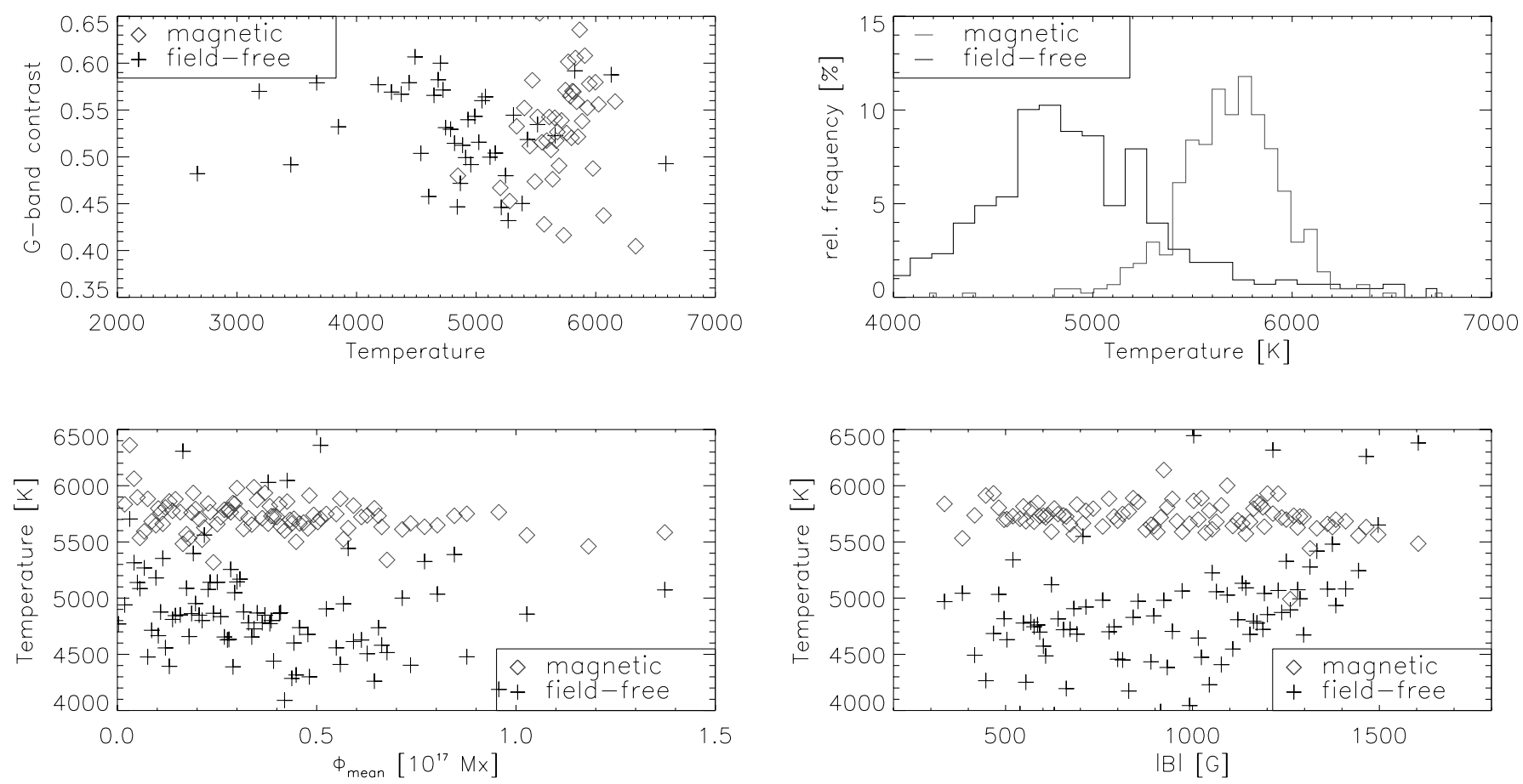

Fig. 18. Top left: G-band contrast vs temperature of the two atmospheric components used to invert the BP profiles: the magnetic interior $(\diamond)$ and the external, field-free surroundings (+). Top right: histogram of temperatures. The magnetic interior is usually hotter than the surroundings by about $1000 \mathrm{~K}$ at the same optical depth. Bottom left: temperature of the two atmospheric components vs. average unsigned magnetic flux. The magnetic temperature is seen to decrease linearly with flux. Bottom right: temperature as a function of field strength. A slight decrease in the magnetic temperature is observed. For the field-free component, no clear trends with $\Phi_{\text {mean }}$ or $B$ exist.

- The distribution of magnetic field inclinations to the LOS, hence to the solar surface, indicates that not all BP fields are vertical.

- The BPs observed in a sunspot moat exhibit redshifts in the magnetized plasma. The Stokes $V$ zero-crossing velocity derived from the IR lines is around $500 \mathrm{~m} \mathrm{~s}^{-1}$, while that derived from the visible lines is approximately $400 \mathrm{~m} \mathrm{~s}^{-1}$. The magnetic component of the inversion shows a redshift of $260 \mathrm{~m} \mathrm{~s}^{-1}$ on average. Moreover, the profiles of visible and IR lines exhibit amplitude and area asymmetries that increase with the magnetic velocity.

- The magnetic interior of BPs is about $1000 \mathrm{~K}$ hotter than the field-free surroundings at equal optical depth. The temperature of the magnetic component is slightly reduced for large magnetic fluxes.

- The area covered by individual BPs in the DOT filtergrams is roughly consistent with the size of BPs inferred from the inversion of the visible and infrared lines. The average effective diameter of BPs is around 100 to $150 \mathrm{~km}$, with few structures larger than $300 \mathrm{~km}$.

For the relations between G-band intensity and magnetic properties of the flux concentrations, we find that:

- The G-band contrast of BPs increases slightly with the magnetic field strength.

- The contrast only slightly depends on the magnetic flux per pixel. It increases with flux for values below $5 \times 10^{16} \mathrm{Mx}$ and decreases with flux for values above $10^{17} \mathrm{Mx}$.

- The G-band contrast of BPs scales with the inclination: the smaller the angle between the magnetic field and the line of sight, the higher the G-band contrast.
Most of these observational findings agree with the expected properties of small-scale magnetic flux elements. First, magnetic elements in horizontal temperature equilibrium have lower gas densities, in order to maintain lateral pressure balance with the external field-free medium. Due to the reduced gas density, a given optical depth corresponds to lower geometrical heights in the magnetic interior than in the field-free surroundings: inside the magnetic flux concentration, deeper and hence hotter layers are seen. The downward shift of the optical depth scale depends on the magnetic field strength, which enters the pressure balance equation (Spruit 1976; Steiner \& Stenflo 1990; Bellot Rubio et al. 2000; Schüssler et al. 2003; Shelyag et al. 2004). Second, a G-band BP essentially outlines a deficit in the abundance of the $\mathrm{CH}$ molecule. Under thermodynamic equilibrium conditions, the amount of $\mathrm{CH}$ in the solar atmosphere depends both on the gas density and on the temperature (Sánchez Almeida et al. 2001b; Steiner et al. 2001; Langhans et al. 2002). A strong enhancement of the G-band intensity contrast with respect to nearby continuum wavelengths indicates a weakening of the $\mathrm{CH}$ lines due to increased thermal dissociation.

We want to explicitly point out the relation between some of our observational findings and the modeling of flux concentrations. The temperature difference between the magnetic and field-free components of the inversion at the same optical depth (Fig. 18) agrees with a shift of the optical depth scale in the presence of magnetic fields. The G-band contrast is found to increase by around 0.2 for fields from 0.4 to $1.5 \mathrm{kG}$, suggesting a stronger shift of the optical depth scale for higher field strengths. However, the trend falls short of the predictions from simulations (e.g. Shelyag et al. 2004, their Fig. 4), which suggest a contrast increase greater than 0.5 over the same range. 

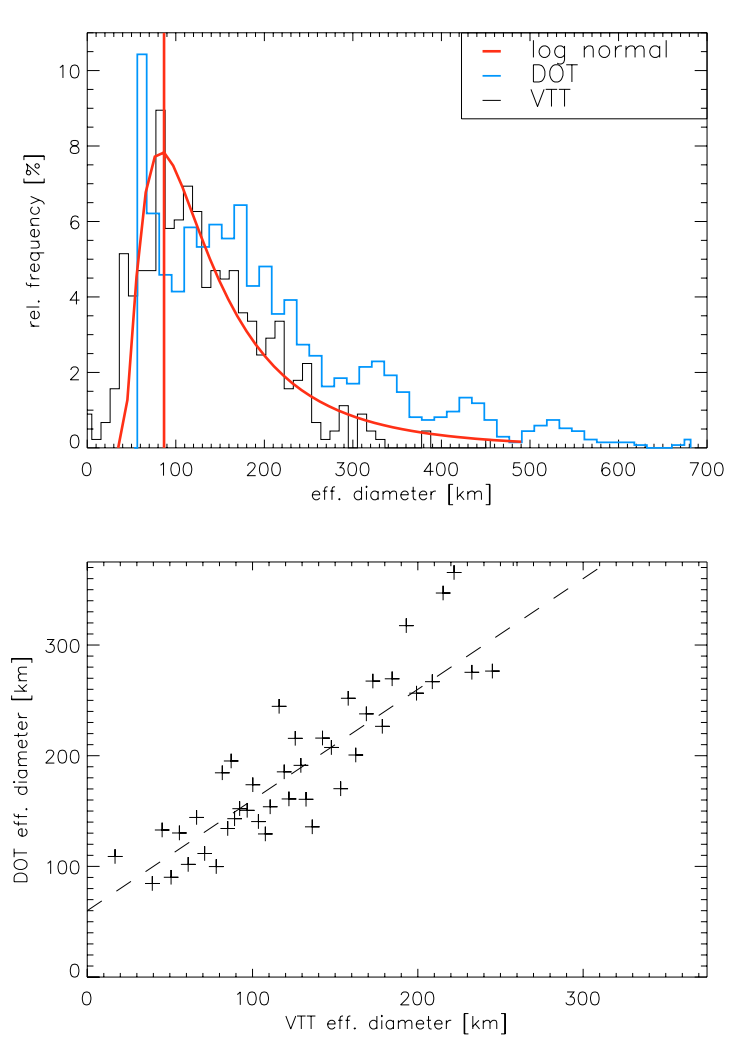

Fig. 19. Top: histogram of BP effective diameters from the VTT (thin black line) and the DOT (thick line). Overplotted is a log-normal distribution. Bottom: effective diameters from the DOT vs. those from the VTT. The dashed line has unity slope and $60 \mathrm{~km}$ offset.

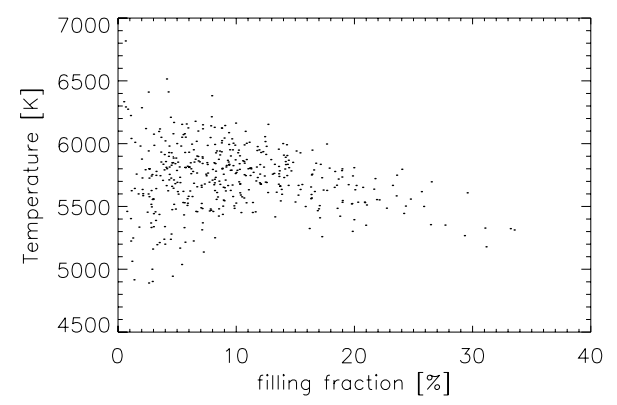

Fig. 20. Temperature of the magnetic atmosphere vs. its filling fraction. For large filling factors, a reduction of the temperature is seen.

The slight decrease in the G-band contrast for magnetic fluxes larger than $10^{17} \mathrm{Mx}$ is at first not predicted by models of small flux elements. However, we suggest that it is actually related to a temperature effect. Since there is an upper limit to the field strength on the order of $1.5 \mathrm{kG}$ (cf. Fig. 8), the amount of flux must be proportional to the diameter of the flux concentration. The volume inside the flux element to be heated increases faster with the diameter of the flux concentration $\left(\propto r^{2} \mathrm{~d} h\right)$ than does the interface with its surroundings $(\propto r \mathrm{~d} h)$. Thus, larger magnetic flux should lead to larger areas, hence to reduced temperatures (Spruit 1976). While this argument is rather indirect, the inversion results allow us to directly check the relation between temperature and area. Figure 20 displays a scatter plot of the temperature of the magnetic component of the inversion vs. the magnetic filling fraction, which is proportional to the area.

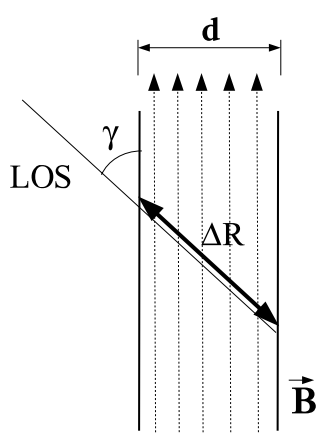

Fig. 21. Sketch of an isolated flux concentration embedded in field-free surroundings. The magnetic field is along the vertical direction and the LOS is inclined by an angle $\gamma$ with respect to it. The path length of a ray inside the flux concentration, $\Delta R$, depends on both $\gamma$ and the thickness $d$ of the tube.

In this plot, we see a clear trend toward a reduction of the temperature with filling factor, hence with area.

Perhaps the most surprising result of our analysis is the dependence of the G-band contrast on the magnetic field inclination to the LOS displayed in the middle panels of Fig. 11. We note that the dependence seems to have the form

$C=a+\frac{b}{\sin \gamma}$

where $\gamma$ represents the field inclination and $a$ and $b$ are constant parameters. A very simple model of a vertical flux concentration embedded in field-free surroundings (Fig. 21) shows that the second term on the right hand side of Eq. (11) is proportional to $\Delta R$, the distance inside the flux concentration traveled by a ray that hits the magnetic element at an angle $\gamma$. In fact, $\Delta R=d / \sin \gamma$. The absorption along the LOS would then be $\propto \Delta R \cdot \rho(B)$, with the density $\rho(B)$ inside the flux concentration being lower than the density outside it. Thus, $\Delta R$ would finally translate into a downward shift of the optical depth scale for lines of sight that pass through the magnetic element, compared with others that miss it. Note that $\Delta R$ is limited by a $\Delta R_{\max }$, the depth where the atmosphere gets opaque even for $\gamma=0 \mathrm{deg}$. We have overplotted three curves for different values of $\mathrm{a}$ and $\mathrm{b}$ in the middle panels of Fig. 11 , where the middle curve $(b=200)$ at least roughly agrees with the observed inclinations and L/V-values.

\section{Conclusions}

We have derived the thermal, magnetic, and kinematic properties of G-band bright points in the moat of a regular sunspot from infrared and visible spectral lines observed at the German Vacuum Tower Telescope on Tenerife. The BPs were identified in cospatial diffraction-limited filtergrams taken with the Dutch Open Telescope on La Palma. The Stokes profiles of the infrared and visible spectral lines were inverted simultaneously using a two-component model atmosphere with height-independent magnetic fields and line-of-sight (LOS) velocities. In addition, line parameters were extracted from the observed Stokes profiles for an estimate of field strengths, field inclinations, and LOS velocities.

We conclude that G-band brightenings are caused by concentrated magnetic fields in more than $90 \%$ of the cases. The minimum requirement seems to be a field strength of at least $500 \mathrm{G}$. However, the G-band BPs show a variety of magnetic and kinematic properties, as suggested by the broad range of values 
we find in all physical quantities $(B, \gamma, \Phi$, etc.). Several patches of magnetic flux can be traced in the polarimetric data during the full 1-hour time series, which show up as BP only part of the time. Together with the broad range of field strengths, we think this indicates that not all BPs are cospatial with stable $k G$ flux tubes. We find a stronger dependence of the G-band brightness on a geometrical effect, namely on the inclination of the magnetic field to the LOS, than on most of the other physical quantities. We conclude that for an accurate description of the generation of G-band BPs or, more generally, the intensity in the G-band, it is necessary to develop models of flux concentrations and their surroundings in at least two dimensions, to take into account the geometry of the field, its strength, and the actual viewing angle of the observations.

Even if our data corresponds to the moat of a sunspot, we believe that the magnetic properties of the observed BPs are not strongly influenced by the presence of the spot. The distance to the spot boundary is several arcseconds in most cases. Thus, the results of this paper might also apply to BPs observed in other active and non-active regions.

Finally, we stress that the use of G-band brightness enhancements as proxies of magnetic fields may miss part of the solar magnetic flux, as fields with strengths below $500 \mathrm{G}$ remain undetected.

Acknowledgements. Discussions with O. Steiner and A. Tritschler are gratefully acknowledged. This work has been supported by the German DFG under grant SCHL 514/2-1 and by the Spanish MEC under Programa Ramón y Cajal and project ESP2003-07735-C04-03. The DOT is operated by Utrecht University at the Spanish Observatorio del Roque de los Muchachos of the Instituto de Astrofísica de Canarias (IAC). The VTT is operated by the Kiepenheuer-Institut für Sonnenphysik at the Spanish Observatorio del Teide, also of the IAC. We thank the referee, Dr. J. Sánchez Almeida, for his suggestions for improving the paper.

\section{References}

Amer, M. A., \& Kneer, F. 1993, A\&A, 273, 304

Auer, L. H., \& Heasley, J. N. 1978, A\&A, 64, 67

Ballesteros, E., Collados, M., Bonet, J. A., et al. 1996, A\&AS, 115, 353

Balthasar, H., Schleicher, H., Bendlin, C., \& Volkmer, R. 1996, A\&A, 315, 603

Beck, C., Schlichenmaier, R., Collados, M., Bellot Rubio, L., \& Kentischer, T. 2005a, A\&A, 443, 1047

Beck, C., Schmidt, W., Kentischer, T., \& Elmore, D. 2005b, A\&A, 437, 1159

Bellot Rubio, L. R., Ruiz Cobo, B., \& Collados, M. 2000, ApJ, 535, 489

Berger, T. E., \& Title, A. M. 1996, ApJ, 463, 365

Berger, T. E., \& Title, A. M. 2001, ApJ, 553, 449
Bonet, J. A., Márquez, I., Muller, R., Sobotka, M., \& Roudier, T. 2005, A\&A, 430, 1089

Borrero, J. M., \& Bellot Rubio, L. R. 2002, A\&A, 385, 1056

Bovelet, B., \& Wiehr, E. 2003, A\&A, 412, 249

Carlsson, M., Stein, R. F., Nordlund, A., \& Scharmer, G. B. 2004, ApJ, 610, L137

Dunn, R. B., \& Zirker, J. B. 1973, Sol. Phys., 33, 281

Gingerich, O., Noyes, R. W., Kalkofen, W., \& Cuny, Y. 1971, Sol. Phys., 18, 347 Grossmann-Doerth, U., Keller, C. U., \& Schuessler, M. 1996, A\&A, 315, 610 Keller, C. U. 1992, Nature, 359, 307

Khomenko, E. V., Collados, M., Solanki, S. K., Lagg, A., \& Trujillo Bueno, J. 2003, A\&A, 408, 1115

López Ariste, A. 2002, ApJ, 564, 379

Landi Degl'Innocenti, E. 2003, AN, 324, 393

Langhans, K., Schmidt, W., \& Tritschler, A. 2002, A\&A, 394, 1069

Langhans, K., Schmidt, W., \& Rimmele, T. 2004, A\&A, 423, 1147

Lites, B. W. 2002, ApJ, 573, 431

Martínez Pillet, V., Lites, B., \& Skumanich, A. 1997, ApJ, 474, 810

Martínez Pillet, V., Collados, M., Sánchez Almeida, J., et al. 1999, in High Resolution Solar Physics: Theory, Observations, and Techniques, ASP Conf. Ser. 183, 264

Mehltretter, J. P. 1974, Sol. Phys., 38, 43

Muller, R. 1983, Sol. Phys., 85, 113

Press, W. H., Flannery, B. P., \& Teukolsky, S. A. 1986, Numerical recipes. The art of scientific computing (Cambridge: University Press)

Reardon, K. P. 2006, Sol. Phys., 239, 503

Rezaei, R., Schlichenmaier, R., Beck, C., \& Bellot Rubio, L. R. 2006, A\&A, 454, 975

Ruiz Cobo, B., \& del Toro Iniesta, J. C. 1992, ApJ, 398, 375

Sütterlin, P., Bellot Rubio, L. R., \& Schlichenmaier, R. 2004, A\&A, 424, 1049

Sanchez Almeida, J. 1998, ApJ, 497, 967

Sanchez Almeida, J., Collados, M., \& del Toro Iniesta, J. C. 1989, A\&A, 222, 311

Sánchez Almeida, J., Asensio Ramos, A., Trujillo Bueno, J., \& Cernicharo, J. 2001a, ApJ, 555, 978

Sánchez Almeida, J., Asensio Ramos, A., Trujillo Bueno, J., \& Cernicharo, J. 2001b, ApJ, 555, 978

Schüssler, M., Shelyag, S., Berdyugina, S., Vögler, A., \& Solanki, S. K. 2003, ApJ, 597, L173

Schmidt, W., \& Kentischer, T. 1995, A\&AS, 113, 363

Schmidt, W., Beck, C., Kentischer, T., Elmore, D., \& Lites, B. 2003, Astron. Nachr., 324, 300

Shelyag, S., Schüssler, M., Solanki, S. K., Berdyugina, S. V., \& Vögler, A. 2004, A\&A, 427, 335

Sigwarth, M., Balasubramaniam, K. S., Knölker, M., \& Schmidt, W. 1999, A\&A, 349,941

Solanki, S. K., \& Pahlke, K. D. 1988, A\&A, 201, 143

Spruit, H. C. 1976, Sol. Phys., 50, 269

Steiner, O., \& Stenflo, J. O. 1990, in IAU Symp., 181

Steiner, O., Hauschildt, P. H., \& Bruls, J. 2001, A\&A, 372, L13

Uitenbroek, H., \& Tritschler, A. 2006, ApJ, 639, 525

van Ballegooijen, A. A., Nisenson, P., Noyes, R. W., et al. 1998, ApJ, 509, 435

Wiehr, E., Bovelet, B., \& Hirzberger, J. 2004, A\&A, 422, L63

Zakharov, V., Gandorfer, A., Solanki, S. K., \& Löfdahl, M. 2005, A\&A, 437, L43 
C. Beck et al.: Magnetic properties of G-band bright points, Online Material p 1

\section{Online Material}




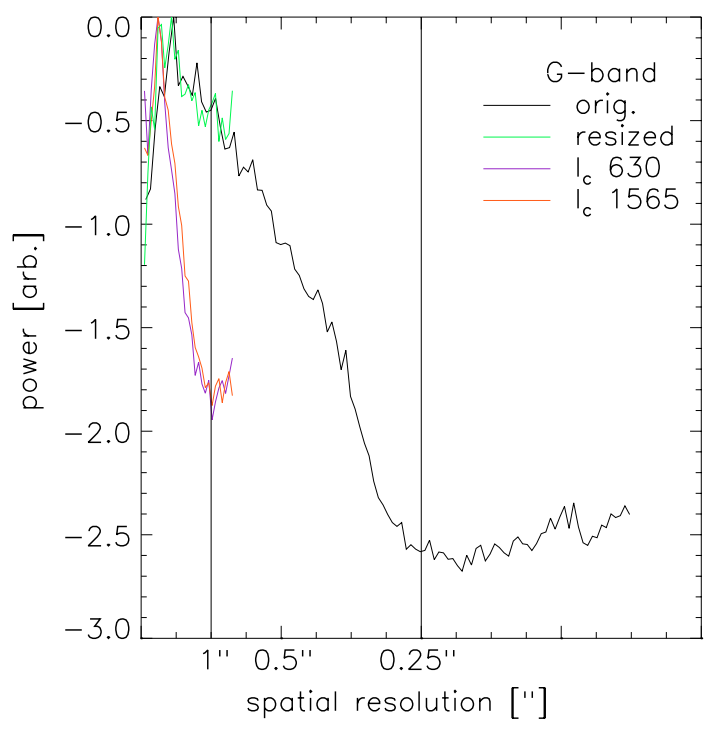

Fig. A.1. Power spectra of the different data sets of the 1st scan as function of spatial resolution.

\section{Appendix A: Spatial resolution}

For an estimate of the spatial resolution, we calculated the Fourier transform of the intensity maps of the first scan across the sunspot. We then integrated the power of the Fourier transform over rings corresponding to increasing spatial frequencies. The inverse of the spatial frequency corresponds to a spatial scale. The resolution limit was estimated as the point, where the power spectrum levels off into the constant noise contribution. For the G-band data from the DOT, we find a resolution of around 0.25 for the original DOT map with 0.071 square pixels (cf. Fig. A.1). When the data is resized to the VTT resolution of 0.37 square pixels, the power is still conserved. For the polarimetric data in either infrared or visible continuum, we find a resolution of 1."0 with this method.

\section{Appendix B: Alignment of data sets}

The alignment procedure uses the TIP pixel as a reference system: for each position $(x, y)_{\text {TIP }}$ in the TIP map, where $x$ corresponds to the scan step and $y$ to the position along the slit, the algorithm looks for cospatial points in the visible and UV channel of POLIS and the DOT filtergrams, respectively. The TIP data have a spatial sampling of 0.35 per pixel. The data from the visible channel of POLIS have a finer sampling of 0.145 , which is degraded to the TIP resolution by the linear interpolation scheme described in the next section. The data from the VTT show displacements due to the actual position of the TIP and POLIS cameras and an additional variable offset caused by differential refraction (e.g. Reardon 2006) in the Earth's atmosphere.

\section{B.1. Alignment between IR and visible/UV data}

Calculation of shifts. To find cospatial points in the TIP and POLIS data, the continuum intensity maps of each repetition of the scan are correlated separately. For the UV channel, a map of the line wing intensity of $\mathrm{Ca}$ II $\mathrm{H}$ is used, as it shows photospheric structure (cf. Fig. 1). The resulting shift values along and
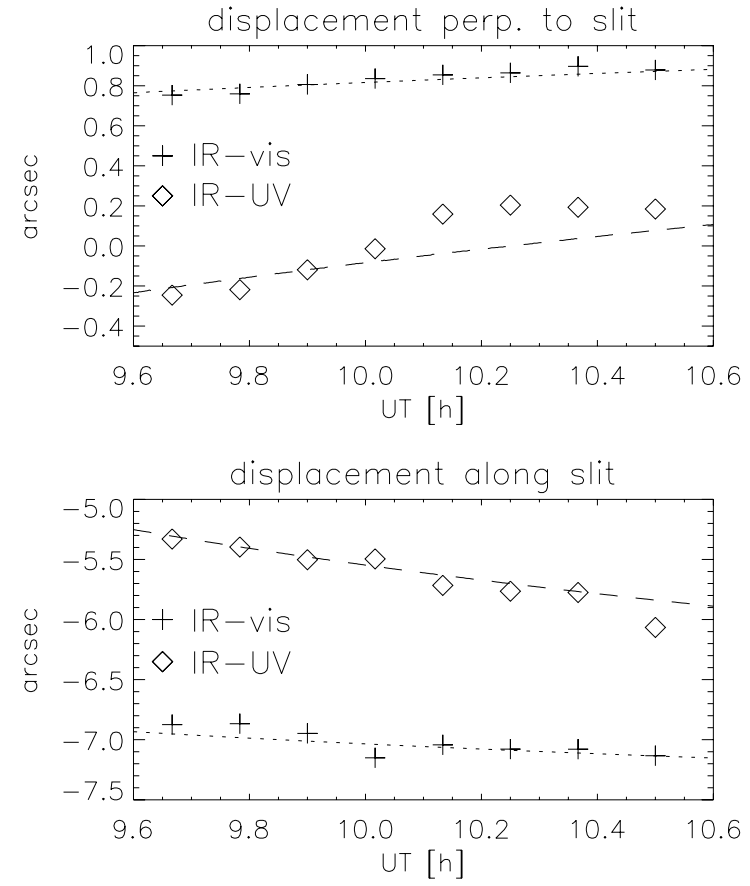

Fig. B.1. Displacements between the visible and infrared intensity maps $(+)$, and between the UV and infrared intensity maps $(\diamond)$, in arcsec. For each repetition of the scan across the spot displayed in Fig. 1, the shifts perpendicular to the slit $(\Delta x ;$ top $)$ and along the slit $(\Delta y ;$ bottom $)$ have been calculated through cross correlation. The dotted and dashed lines give the shift values used for the alignment of each scan step.

perpendicular to the slit for the different times of the eight repetitions allow a curve to be fitted to the displacements (Fig. B.1). The best-fit curve is used to determine the shifts in $x$ and $y$ needed to align the TIP and POLIS observations for each scan step (taken every $6 \mathrm{~s}$ ).

Application of shifts. The spatial sampling of 0.'29 in the UV channel of POLIS is similar to that of TIP. For this reason, the shift $\left(\Delta x_{\mathrm{UV}}, \Delta y_{\mathrm{UV}}\right)$ is simply added to the position $(x, y)_{\mathrm{TIP}}$, and the closest pixel is taken:

$$
(x, y)_{\mathrm{UV}}=\operatorname{round}\left[(x, y)_{\mathrm{TIP}}+\left(\Delta x_{\mathrm{UV}}, \Delta y_{\mathrm{UV}}\right)\right],
$$

where the rounding automatically selects the nearest cospatial pixel.

The spatial sampling in the visible channel of POLIS is almost three times better than that of TIP. The cospatial Stokes profiles are then retrieved as a weighted average of the visible profiles along and perpendicular to the slit. First, the cospatial position $(x, y)_{\text {vis }}$ is given by

$(x, y)_{\mathrm{vis}}=(x, y)_{\mathrm{TIP}}+\left(\right.$ floor$\left.\left[\Delta x_{\mathrm{vis}}\right], \Delta y_{\mathrm{vis}}\right)$,

where floor $\left[\Delta x_{\text {vis }}\right]$ returns the largest integer smaller than $x_{\text {vis }}$.

The TIP pixel partly covers several POLIS pixels from two different scan steps, $x_{\text {vis }}$ and $x_{\text {vis }}+1$ (Fig. B.2). The cospatial Stokes profile $p(\lambda, x, y)_{\text {vis }}$ is calculated as the weighted average

$p(\lambda, x, y)_{\mathrm{vis}}=\frac{\sum_{i} a_{i} p\left(\lambda, x, y_{i}\right)_{\mathrm{vis}}}{\sum_{i} a_{i}}+\frac{\sum_{i} b_{i} p\left(\lambda, x+1, y_{i}\right)_{\mathrm{vis}}}{\sum_{i} b_{i}}$,

where $i=1, \ldots, 3$ or $i=1, \ldots, 4$. The weights $a_{i}$ and $b_{i}$ are set according to the fraction of the POLIS pixels covered by the TIP pixel and verify the condition $\sum_{i}\left(a_{i}+b_{i}\right)=1$. 


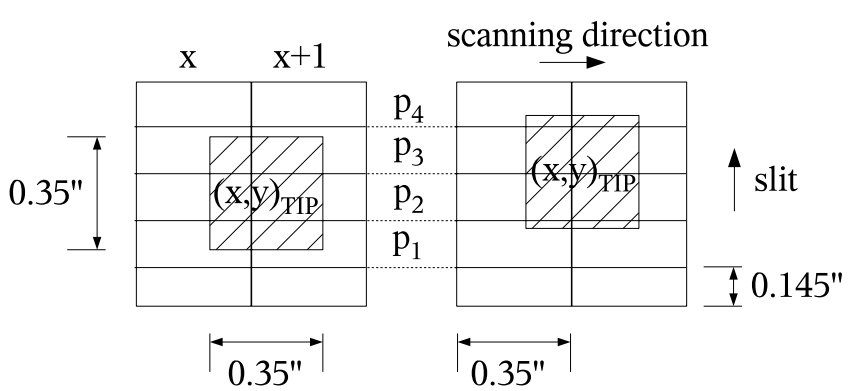

Fig. B.2. Calculation of the visible profile that is cospatial to the infrared Stokes profile at $(x, y)_{\text {TIP. }}$ Two cases are possible: the TIP pixel covers three (left) or four (right) POLIS pixels along the slit. The profiles $p_{i}$ from the two scan steps $x_{\mathrm{vis}}$ and $x_{\mathrm{vis}}+1$ are averaged with weights corresponding to the fraction of the POLIS resolution elements covered.

\section{B.2. Alignment between DOT and VTT data}

In this case, cospatial and cotemporal positions have to be found. A cotemporal map from the DOT time series ("pseudo-scan") is first constructed and then spatially aligned with the VTT data.

\section{B.2.1. Cotemporal DOT map}

The DOT pseudo-scan is the image that would result from stepping the POLIS/TIP slit across the DOT FOV. To create this map, the POLIS slit-jaw images are used. They display a $100^{\prime \prime} \times 100^{\prime \prime}$ FOV of the solar surface centered on the slit. Each slit-jaw image is correlated with the DOT blue continuum filtergram closest in time, degraded to the same spatial resolution. From the correlation one finds the shifts in $x$ and $y$ required to align the images from the two telescopes. The POLIS slit visible in the slitjaw image is superimposed on the coaligned DOT filtergram. A slice of 5 pixel width $(=0.355)$ is taken from the DOT images at the slit position and placed accordingly in the pseudo-scan map. Examples of the resulting pseudo-scan maps for the three DOT channels are displayed in Fig. 1.

\section{B.2.2. Cospatial DOT map}

The final alignment of the DOT images is achieved by taking a $25 \times 25$ pixel subfield of the TIP map around each pixel $(x, y)_{\mathrm{TIP}}$, the corresponding area from the DOT pseudo-scan continuum map, and correlating the two subfields. This procedure yields the position of a $5 \times 5$ pixel area in the DOT maps that is cospatial with the TIP pixel. The area is cut out from the three DOT pseudo-scan maps and placed in new maps accordingly. Figure 3 displays the coaligned maps for the first repetition of the scan. The flow chart displayed in Fig. B.3 summarizes all the steps we follow to align the various data sets.

\section{Appendix C: Coaligned maps}

Coaligned DOT and VTT maps are displayed in Figs. C.1 and C.2 for the eight repetitions of the scan. We show the aligned DOT G-band, the total integrated polarization, the IR continuum intensity, and the DOT BP mask. For the first repetition of the scan, the VTT mask of identified BPs is displayed instead of the DOT BP mask. The area used to extract the NBP sample is also indicated for the first repetition. A visual inspection shows that almost all features identified as G-band bright points (red contours) are cospatial with polarization signals above the noise. A single polarization patch usually contains a few BPs, and the

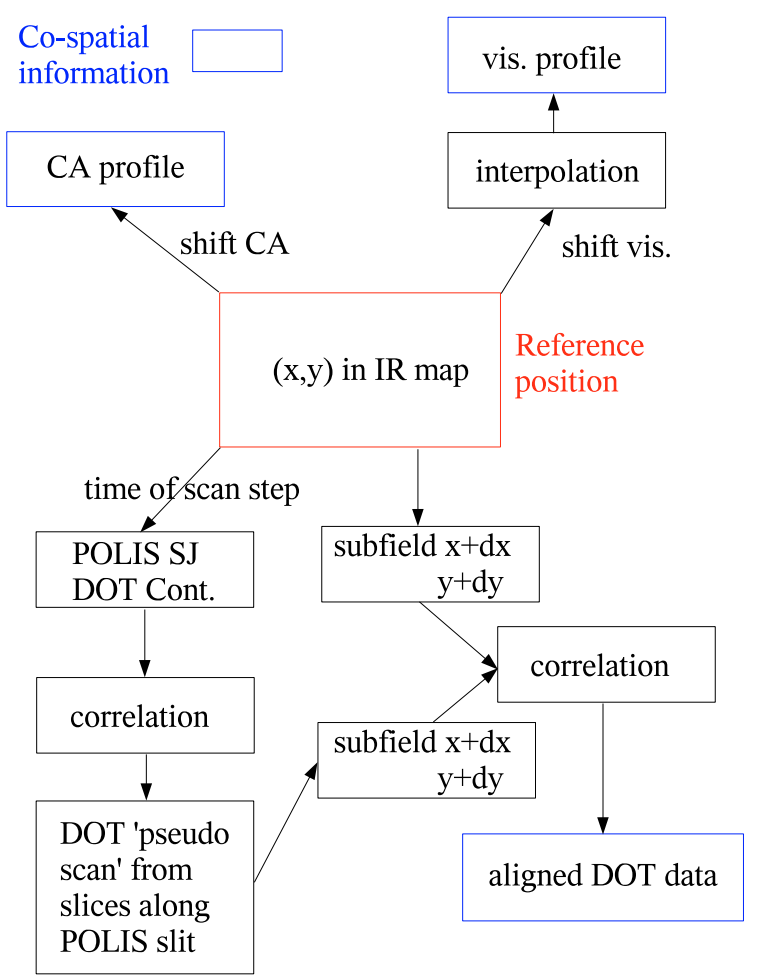

Fig. B.3. Alignment procedure. For each position $(x, y)_{\mathrm{TIP}}$ in the TIP data set, cospatial POLIS profiles are found by applying appropriate shifts to the visible and UV channels. To align the DOT filtergrams, the POLIS slit-jaw image and the DOT continuum image closest in time are correlated and used for the creation of a pseudo-scan map. The final DOT/VTT alignment is done by taking a subfield of the TIP map around $(x, y)_{\text {TIP }}$, the corresponding subfield from the DOT pseudo-scan, and then correlating the subfields.

majority of polarization patches show BPs at least in one of the repetitions. Most magnetic signals remain visible during the full observation run $(1 \mathrm{~h})$. This agrees with the interpretation that brightenings in the $\mathrm{G}$ band are due to the presence of magnetic fields, while the high temporal variability of the BPs is due to changes in the magnetic field configuration.

The sunspot canopy (visible near the upper edge of the FOV) clearly extends beyond the white-light boundary of the spot. The canopy is not an area of enhanced G-band intensity because its magnetic fields are very inclined and do not lead to a sufficient downward shift of the optical depth scale. 
C. Beck et al.: Magnetic properties of G-band bright points, Online Material p 4
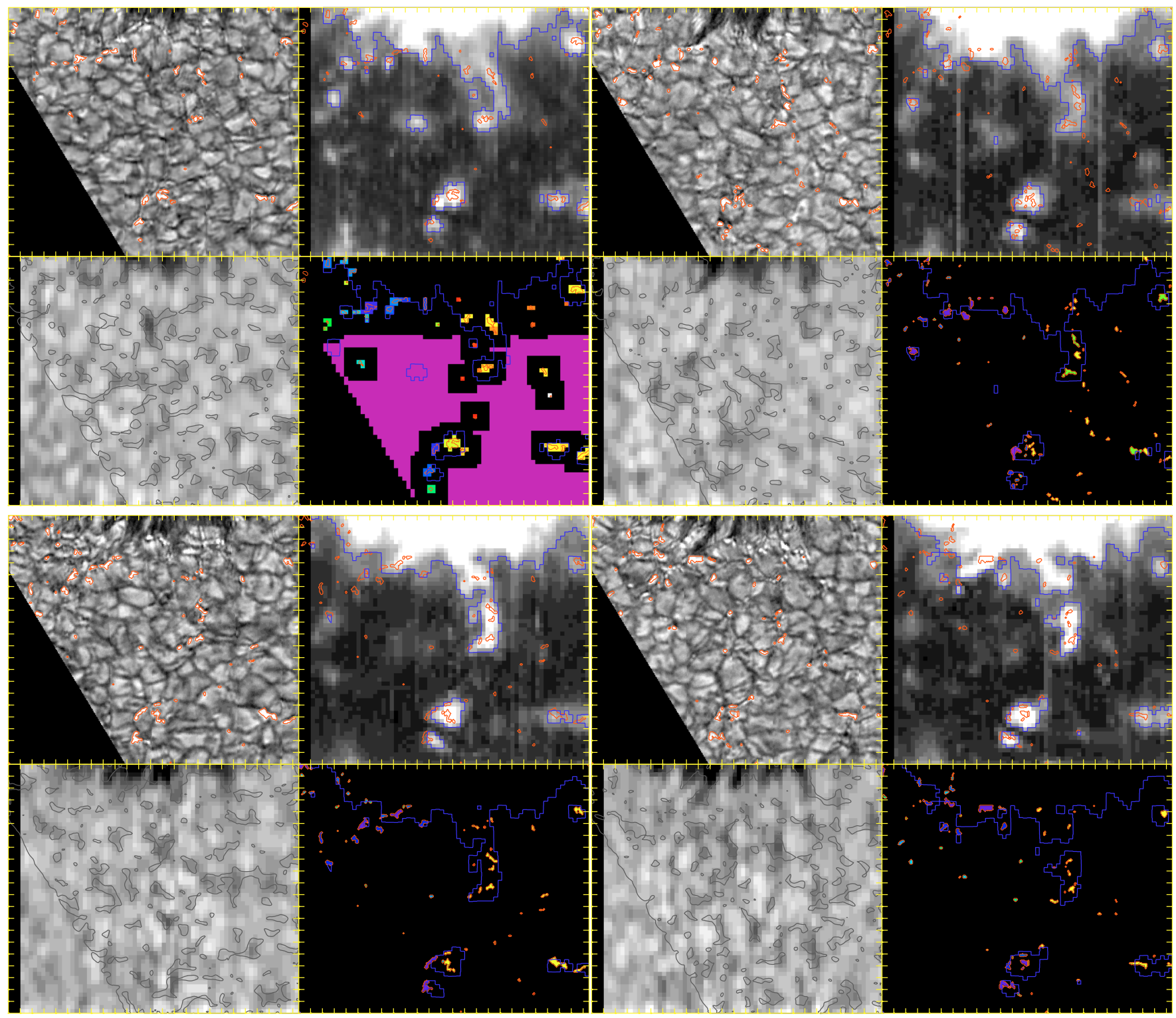

Fig. C.1. Coaligned maps from the VTT and the DOT for the first four repetitions of the scan. Clockwise, starting from top left of each $4 \times 4$ subpanel: G-band intensity, total integrated polarization, DOT BP mask, and IR continuum intensity. Blue contours outline enhanced polarization signals and red contours the selected BP areas. In the DOT BP mask, the BPs are color-coded according to their number. The lower right map of the first $4 \times 4$ subpanel is the VTT BP mask, not the DOT BP mask. In this map, the uniform pink area marks the spatial points used to extract the NBP sample. They are located outside the canopy, and at least 3 pixels (1".05) away from any BP. 
C. Beck et al.: Magnetic properties of G-band bright points, Online Material p 5

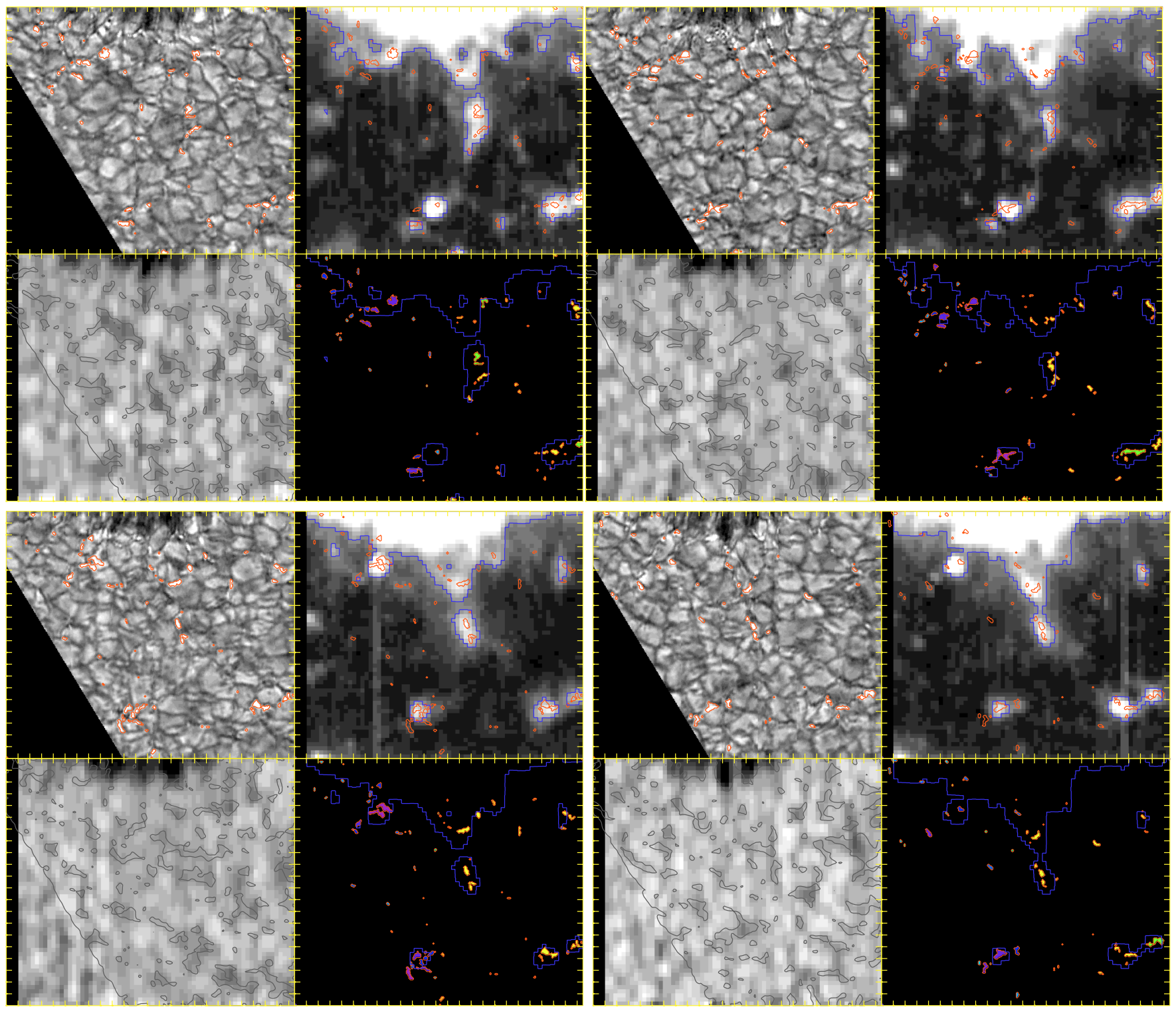

Fig. C.2. Same as Fig. C.1, for the last four repetitions of the scan. 